\title{
Tidal effects in the equations of motion of compact binary systems to next-to-next-to-leading post-Newtonian order
}

\author{
Quentin Henry,* Guillaume FAYe, ${ }^{\dagger}$ and Luc BlancheT ${ }^{\ddagger}$ \\ $\mathcal{G} \mathbb{R} \varepsilon \mathbb{C O}$, Institut d'Astrophysique de Paris, \\ UMR 7095, CNRS, Sorbonne Université, \\ $98^{\text {bis }}$ boulevard Arago, 75014 Paris, France
}

(Dated: November 2, 2020)

\begin{abstract}
As a first step in the computation of the orbital phase evolution of spinless compact binaries including tidal effects up to the next-to-next-to-leading (NNL) order, we obtain the equations of motion of those systems and the associated conserved integrals in harmonic coordinates. The internal structure and finite size effects of the compact objects are described by means of an effective Fokker-type action. Our results, complete to the NNL order, correspond to the second-post-Newtonian $(2 \mathrm{PN})$ approximation beyond the leading tidal effect itself, already occurring at the 5PN order. They are parametrized by three polarizability (or deformability) coefficients describing the mass quadrupolar, mass octupolar and current quadrupolar deformations of the objects through tidal interactions. Up to the next-to-leading (NL) order, we recover previous results in the literature; up to the NNL order for quasi-circular orbits, we confirm the known tidal effects in the (PN re-expansion of the) effective-one-body (EOB) Hamiltonian. In a future work, we shall derive the tidal contributions to the gravitational-wave flux up to the NNL order, which is the second step required to find the orbital phase evolution.
\end{abstract}

\footnotetext{
* henry@iap.fr

$\dagger$ faye@iap.fr

‡ blanchet@iap.fr
} 


\section{INTRODUCTION}

The direct detection of gravitational waves $(\mathrm{GW})$ generated by the orbital motion and merger of compact binary systems [1, 2] opens up a new avenue in fundamental physics. Notably, it will play a paramount role in understanding the physics of compact objects, mainly black holes or neutron stars. The tidal effects between such objects are particularly interesting because they permit revealing and probing their internal structure, as well as eventually distinguishing between black holes, neutron stars or, possibly, more exotic entities like boson stars [3, 4].

The tidal interaction affects both the conservative equations of motion (EoM) and the GW emission of the compact binary system. This results in a modification of the time evolution of the binary's orbital frequency and phase which is directly observable (see e.g. [5-8]). The tidal distortion depends on the Love numbers [9], characterizing the rigidity and the deformability of the body, i.e. its capacity to change shape under the influence of an external tidal field. Those Love numbers depend in turn on the internal equation of state (EoS) of the body, which is uncertain at high densities [10,11]. They decrease as the compactness of the body increases, reaching zero in the limit of a maximally compact object, i.e., for a black hole [12-14].

The leading tidal contributions to the orbital dynamics are due to quadrupolar deformations and, for compact binaries, manifest themselves as formally very small corrections in the accelerations, of the order of $5 \mathrm{PN}$ or $\sim(v / c)^{10}$, where $v$ denotes the relative orbital velocity. However, the $5 \mathrm{PN}$ coefficient appearing in front of the small $5 \mathrm{PN}$ factor $(v / c)^{10}$ can be quite large and the effect is measurable. ${ }^{1}$ It scales like the dimensionless parameter

$$
\Lambda^{(2)}=\frac{2}{3} k^{(2)}\left(\frac{R c^{2}}{G m}\right)^{5},
$$

where $k^{(2)}$ denotes the mass-type quadrupolar second Love number of the body, while $m$ and $R$ represent its mass and radius. Typically, the compactness parameter $\mathcal{C} \sim G m /\left(R c^{2}\right)$ is of order 0.15 for neutron stars while the Love number is $k^{(2)} \sim 0.1$ (depending on the EoS) [13, 14], hence we expect $\Lambda^{(2)} \sim 1000$. With the binary neutron star event GW170817 [2], the detectors LIGO and Virgo have already been able to put an observational constraint on the particular combination of $\Lambda_{1}^{(2)}, \Lambda_{2}^{(2)}$ and the masses that enter the orbital phase evolution of the two neutron stars [6, 8]. This constraint permitted excluding some of the stiffest EoS, for which the neutron stars are less compact [15, 16]. However, the majority of softer EoS are still allowed (see also [17] and references therein).

The problem of tidal interactions between compact objects beyond the leading quadrupolar level has been addressed in Refs. [18-23]. The conservative dynamics, from which follow the EoM, was obtained in the work [21] at leading order but including linear spin couplings, or in [20] and [19] up to the next-to-leading (NL) and the next-to-next-to-leading (NNL) orders, respectively, while the energy flux, waveform amplitude and phase evolution have been computed to the leading order in the presence of spin couplings, and NL order, equivalent to the formal 6PN level [18, 22, 23], in the non-spinning case. ${ }^{2}$ The tidal interactions in both the dynamics and waveform have also been included in the effective-one-body (EOB) models for template generation [7, 19].

In the present paper, we compute the tidal effects in the conservative EoM, as well as all associated conserved quantities, at the NNL order for spinless neutron stars on generic binary orbits in harmonic coordinates. We follow closely the method proposed in Ref. [19], describing the internal structure and finite size effects of the compact objects by means of an effective Fokker-type action. Our final NNL results are parametrized by three polarizability (or deformability) coefficients describing the mass quadrupolar, mass octupolar and current quadrupolar deformations of the objects through tidal interactions. In the case of quasi-circular orbits, we confirm the expression of the tidal terms in the EOB Hamiltonian up to the NNL order [19]. So as to compute the tidal contribution to the orbital phase at the NNL order, we need both the conservative NNL energy of the system and the GW energy flux at the same NNL order. In a forthcoming paper [24], we shall complete the present work by computing the latter effect for the GW flux, which will yield the orbital phase evolution at the NNL order.

Although the knowledge of the NNL/2PN relative tidal effect is probably not directly useful for the data analysis of the advanced LIGO and Virgo detectors, it may become relevant for the future third-generation detectors, like the Einstein Telescope or the Cosmic Observatory. On the other hand, detailed comparisons with numerical relativity (NR) simulations of binary neutron-star mergers require the control of high-order tidal interactions on the analytic side. Yet, such comparisons are essential to get a grip on the errors of the predicted waveforms and to properly calibrate EOB models. More generally, adding analytic tidal effects on the top of PN templates of point particles is a good way of controlling the systematic errors due to our lack of knowledge of the higher-order terms in the PN expansion $[4,8]$.

\footnotetext{
${ }^{1}$ One can speculate that the tidal 5PN coefficient is larger than the purely orbital 5PN contribution to the orbital phase for point particles, which is currently unknown.

${ }^{2}$ The NNL order in the dynamics corresponds to $2 \mathrm{PN}$ order beyond the leading $5 \mathrm{PN}$ quadrupolar tidal effect and is thus formally equivalent to a $7 \mathrm{PN}$ orbital effect; similarly, the NL order means $1 \mathrm{PN}$ beyond the leading $5 \mathrm{PN}$ effect.
} 
This article is organized as follows. In Sec. II, we define the effective Fokker action with appropriate non-minimal matter couplings describing finite size effects. The quantities entering this action are determined by the $2 \mathrm{PN}$ metric, presented in Sec. III and computed off-shell, i.e., without replacement of accelerations by the EoM, ready for insertion into the action. Our final Lagrangian, accurate to NNL leading order for tidal effects, is displayed in Sec. IV, together with the associated NL center-of-mass (CoM) position. We then derive, in Sec. V, the tidal dynamics in the CoM frame for general orbits, as well as the reduction for quasi-circular orbits. The Appendix A is devoted to basic recalls and motivation concerning the treatment of tidal effects in the Newtonian theory. In Appendix B we show, using standard techniques of Lagrangian formalism, that the tidal multipole moments up to the NNL order can be defined equivalently by means of either the Riemann tensor or the Weyl tensor. Finally we give in Appendix C the complete tidal acceleration in a general frame for arbitrary orbits to NNL order.

\section{EFFECTIVE FOKKER ACTION WITH NON-MINIMAL MATTER COUPLINGS}

The model we use is defined by the gravitation-plus-matter action $S=S_{g}+S_{m}$, where the gravitational part $S_{g}$ is the standard Einstein-Hilbert action, to which we add the appropriate harmonic-gauge fixing term:

$$
S_{g}=\frac{c^{3}}{16 \pi G} \int \mathrm{d}^{4} x \sqrt{-g}\left[R-\frac{1}{2} g_{\mu \nu} \Gamma^{\mu} \Gamma^{\nu}\right],
$$

where $R$ is the curvature scalar, $\Gamma_{\rho \sigma}^{\mu}$ is the usual Christoffel symbol, and $\Gamma^{\mu}=g^{\rho \sigma} \Gamma_{\rho \sigma}^{\mu}$. In practical calculations, we rather use the Laudau-Lifshitz [25] form of the action. ${ }^{3}$

The matter part of the action $S_{m}$ describes massive point-like particles with internal structure. It contains specific non-minimal couplings to the space-time curvature that describe the finite size effects of the compact bodies solely due to the tidal interactions, all spins being taken to zero. Since the matter action is regarded as localized on the worldline of the particles, it is generally referred to as a "skeletonized" effective action. In order to define it, we introduce a local inertial coordinate frame along each body worldline, together with the associated local tetrad $e_{\hat{\alpha}}{ }^{\mu}$. More precisely, we pose $e_{\hat{\alpha}}{ }^{\mu}=\partial x^{\mu} / \partial X^{\hat{\alpha}}$, where $\left\{x^{\mu}\right\}$ is a global coordinate system and $\left\{X^{\hat{\alpha}}\right\}$ is the local inertial frame in the vicinity of the body in question. We may choose $\left\{X^{\hat{\alpha}}\right\}$ to be a Fermi local normal coordinate system [27, 28], so that the tetrad is orthonormal on the worldline, the time coordinate of the Fermi coordinates coincides with the proper time along the worldline, and the zero-th time-like tetrad vector is the four velocity of the particle. In its own local frame, the body feels the tidal multipole moments generated by the other bodies at its very location, namely the $\ell$-th order mass-type moments $G_{\hat{L}}$ and the current-type ones $H_{\hat{L}}$, where those quantities refer to the spatial tetradic components of the moments, i.e. projected along the local tetrad, with $\hat{L}=\hat{i}_{1} \cdots \hat{i}_{\ell}$ denoting a multi-spatial index composed of $\ell$ spatial tetradic indices.

In this paper, we assume that each body stays in static equilibrium at any instant. In the absence of spin, the internal structure is then entirely determined by the mass and the EoS. Thus, the elementary bricks that are allowed to construct $S_{m}$ are tensors defined from the metric only and evaluated at the given particle position, with all indices contracted so as to preserve the invariance under rotation and parity in the corresponding constant-time hypersurface of the local Fermi rest frame. For our purpose, it will be sufficient to consider the same non-minimal terms as in Ref. [29], built from quadratic (kinetic-like) couplings in the tidal moments $G_{\hat{L}}$ and $H_{\hat{L}}$. Hence the form of the matter action (adding also the particle's label $A \in\{1,2\})^{4}$

$$
S_{m}=\sum_{A} \int \mathrm{d} \tau_{A}\left\{-m_{A} c^{2}+\sum_{\ell=2}^{+\infty} \frac{1}{2 \ell !}\left[\mu_{A}^{(\ell)}\left(G_{\hat{L}}^{A}\right)^{2}+\frac{\ell}{(\ell+1) c^{2}} \sigma_{A}^{(\ell)}\left(H_{\hat{L}}^{A}\right)^{2}\right]+\cdots\right\} .
$$

The ellipsis indicate many higher-order non-linear combinations of the tidal moments and their covariant (propertime) derivatives, which we do not need to include here (see e.g. Eq. (2.3) of [19]). For more insight and motivation about the non-minimal action, see Refs. [19, 30-32] and the treatment of tidal effects in the Newtonian model as recalled in Appendix A.

The above tidal moments are given by appropriate covariant derivatives of the Weyl tensor. We define first the spatial tetradic components of the moments appearing in Eq. (2.2) (for $\ell \geqslant 2$ ) as

$$
G_{\hat{L}}^{A}=-c^{2}\left[\nabla_{\left\langle\hat{i}_{1}\right.} \cdots \nabla_{\hat{i}_{\ell-2}} C_{\left.\hat{i}_{\ell-1} \underline{\hat{0}} \underline{\hat{i}} \ell\right\rangle \hat{0}}\right]_{A},
$$

\footnotetext{
${ }^{3}$ Throughout the paper, we use the conventions of MTW [26]; in particular, the metric signature is $(-,+,+,+)$ and the Riemann tensor satisfies the identity $\left(\nabla_{\mu} \nabla_{\nu}-\nabla_{\nu} \nabla_{\mu}\right) V^{\lambda}=R_{\kappa \mu \nu}^{\lambda} V^{\kappa}$.

${ }^{4}$ The constant mass of body $A$ is denoted $m_{A}$ and its proper time $\mathrm{d} \tau_{A}=\left(-\left[g_{\mu \nu}\right]_{A} \mathrm{~d} y_{A}^{\mu} \mathrm{d} y_{A}^{\nu} / c^{2}\right)^{1 / 2}$, where $y_{A}^{\mu}\left(\tau_{A}\right)$ is the particle's worldline. The four velocity $u_{A}^{\mu}=\mathrm{d} y_{A}^{\mu} /\left(c \mathrm{~d} \tau_{A}\right)$ is such that $\left[g_{\mu \nu}\right]_{A} u_{A}^{\mu} u_{A}^{\mu}=-1$, with $\left[g_{\mu \nu}\right]_{A}$ denoting the metric regularized at the location of body $A$; this is of course nothing but the time-time component of the orthonormalizing condition of the tetrad, $\eta_{\hat{\alpha} \hat{\beta}}=\left[g_{\mu \nu}\right]_{A} e_{\hat{\alpha}}^{A \mu} e_{\hat{\beta}}^{A \nu}$.
} 


$$
H_{\hat{L}}^{A}=2 c^{3}\left[\nabla_{\left\langle\hat{i}_{1}\right.} \cdots \nabla_{\hat{i}_{\ell-2}} C_{\left.\hat{i}_{\ell-1} \underline{\hat{0}} \hat{i} \ell\right\rangle \hat{\jmath}}^{*}\right]_{A} .
$$

The angle brackets over the $\ell$ free spatial indices $\hat{L}=\hat{i}_{1} \cdots \hat{i}_{\ell}$ of the above tensor expressions means that they must be replaced by their symmetric and trace-free (STF) parts over those indices, the underlined indices being excluded from the STF projection. We denote by $\nabla_{\hat{\alpha}}$ the usual covariant tetradic derivative [we pose $\hat{\alpha}=(\hat{0}, \hat{i})$ ], whereas $C_{\hat{\alpha} \hat{\beta} \hat{\gamma} \hat{\delta}}$ and $C_{\hat{\alpha} \hat{\beta} \hat{\gamma} \hat{\delta}}^{*}$ represent the tetradic components of the Weyl tensor (whose definition is recalled in Eq. (B2) below) and its dual. ${ }^{5}$ By construction, the tidal moments (2.3) are symmetric over their spatial indices $\hat{L}$ and all their traces are zero, i.e., $\delta_{\hat{i}_{1} \hat{i}_{2}} G_{\hat{i}_{1} \hat{i}_{2} \ldots \hat{i}_{\ell}}=0$.

Next, we introduce the covariant versions of the previous tidal tensors. Since $u^{\mu}=e_{\hat{0}}{ }^{\mu}$, this is achieved by imposing that they live in the particle's local spatial hypersurface, which is orthogonal to the four velocity. Thus, we complete the definition of the tidal moments (2.3) by requiring them to obey

$$
G_{\hat{0} \hat{\alpha}_{2} \cdots \hat{\alpha}_{\ell}}^{A}=H_{\hat{0} \hat{\alpha}_{2} \cdots \hat{\alpha}_{\ell}}^{A}=0 .
$$

In this way, $G_{\hat{\alpha}_{1} \ldots \hat{\alpha}_{\ell}}$ and $H_{\hat{\alpha}_{1} \ldots \hat{\alpha}_{\ell}}$ are both Lorentz tensors and covariant scalars, while their covariant versions in an arbitrary coordinate system $\left\{x^{\mu}\right\}$ read

$$
\begin{aligned}
& G_{\mu_{1} \cdots \mu_{\ell}}^{A}=-c^{2}\left[\nabla_{\left\langle\mu_{1}\right.}^{\perp} \cdots \nabla_{\mu_{\ell-2}}^{\perp} C_{\left.\mu_{\ell-1} \underline{\rho} \mu_{\ell}\right\rangle \sigma}\right]_{A} u_{A}^{\rho} u_{A}^{\sigma}, \\
& H_{\mu_{1} \cdots \mu_{\ell}}^{A}=2 c^{3}\left[\nabla_{\left\langle\mu_{1}\right.}^{\perp} \cdots \nabla_{\mu_{\ell-2}}^{\perp} C_{\left.\mu_{\ell-1} \underline{\rho} \mu_{\ell}\right\rangle \sigma}^{*}\right]_{A} u_{A}^{\rho} u_{A}^{\sigma} .
\end{aligned}
$$

Here, we denote $\nabla_{\mu}^{\perp}=\perp_{\mu}^{\nu} \nabla_{\nu}$, with $\perp_{\mu}^{\nu}=\delta_{\mu}^{\nu}+u_{\mu} u^{\nu}$ being the projector onto the hypersurface orthogonal to the four velocity [notice that $\perp_{\hat{\alpha}}^{\mu}=\left(0, e_{\hat{i}}^{\mu}\right)$ ]. The tidal moments are both STF over all their space-time indices and transverse to the four velocity, namely $u^{\mu} G_{\mu \mu_{2} \cdots \mu_{\ell}}=u^{\mu} H_{\mu \mu_{2} \cdots \mu_{\ell}}=0$, which is equivalent to (2.4).

Very important to the formalism is the fact that the Weyl tensor and its covariant derivatives in (2.5) are to be evaluated at the location of the particle $A$ following the regularization, as indicated by the square brackets $[\cdots]_{A}$. Physically, the regularization is crucial because it removes the self field of the particle $A$, and therefore permits automatically selecting the external (tidal) field due to the other particles $B \neq A$. We know one regularization able to give a complete, consistent and physical answer in high PN approximations, namely dimensional regularization (see e.g. $[33,34])$. In this paper, we shall systematically use it. However, in our practical calculations at the relatively low NNL/2PN order, it is simpler to use the Hadamard "partie finie" regularization, since it has been shown [19] to yield the same result for the specific system we are interested in (see also discussions in Ref. [34]).

On the other hand, as argued in Refs. [19, 35], we can choose to use, for our purpose, the Riemann tensor instead of the Weyl tensor in the definitions (2.5) of the tidal moments. Indeed, the contributions due to the trace terms of the Riemann tensor may be absorbed in the off-shell metric by redefining it in a certain way. We give in Appendix B a detailed proof of this statement valid up to the NNL/2PN level.

Note finally that the tidal moments (2.5) have been normalized in such a way that they admit a finite non-zero Newtonian limit when $c \rightarrow+\infty$, and that the mass-type moments then match those of Newtonian mechanics given in Appendix A. In this limit, only the space components survive. We then get

$$
\begin{aligned}
& G_{L}^{A}=\partial_{L}^{A} U_{A}+\mathcal{O}\left(\frac{1}{c^{2}}\right), \\
& H_{L}^{A}=4 \varepsilon_{j k\left(i_{\ell}\right.}\left(\partial_{L-1) k}^{A} U_{j}^{A}+v_{A}^{k} \partial_{L-1) j}^{A} U_{A}\right)+\mathcal{O}\left(\frac{1}{c^{2}}\right),
\end{aligned}
$$

where $\partial_{L}^{A}=\partial_{i_{1}}^{A} \cdots \partial_{i_{\ell}}^{A}$ with $\partial_{i}^{A}=\partial / \partial y_{A}^{i}$; the potentials $U_{A}=\sum_{B \neq A} G m_{B} / r_{B}$ and $U_{A}^{i}=\sum_{B \neq A} G m_{B} v_{B}^{i} / r_{B}$ denote the Newtonian and gravitomagnetic potentials regularized at the point $A$.

As the tidal moments are transverse to the velocity, the action (2.2) can be rewritten in covariant form as

$$
S_{m}=\sum_{A} \int \mathrm{d} \tau_{A}\left\{-m_{A} c^{2}+\sum_{\ell=2}^{+\infty} \frac{1}{2 \ell !}\left[\mu_{A}^{(\ell)} G_{\mu_{1} \cdots \mu_{\ell}}^{A} G_{A}^{\mu_{1} \cdots \mu_{\ell}}+\frac{\ell}{(\ell+1) c^{2}} \sigma_{A}^{(\ell)} H_{\mu_{1} \cdots \mu_{\ell}}^{A} H_{A}^{\mu_{1} \cdots \mu_{\ell}}\right]+\cdots\right\} .
$$

${ }^{5}$ In our convention, $C_{\hat{\alpha} \hat{\beta} \hat{\gamma} \hat{\delta}}^{*} \equiv \frac{1}{2} \varepsilon_{\hat{\alpha} \hat{\beta} \hat{\eta} \hat{\zeta}} C_{\hat{\gamma} \hat{\gamma} \hat{\delta}}$ or, in covariant form, $C_{\mu \nu \rho \sigma}^{*} \equiv \frac{1}{2} \varepsilon_{\mu \nu \lambda \kappa} C_{\rho \kappa}^{\lambda \kappa}$, where $\varepsilon_{\hat{\alpha} \hat{\beta} \hat{\gamma} \hat{\delta}}$ denotes the tetradic components of the completely anti-symmetric Levi-Civita tensor $\varepsilon_{\mu \nu \rho \sigma}$, defined by $\varepsilon_{\hat{0} \hat{1} \hat{2} \hat{z}}=1$ and $\varepsilon_{0123}=\sqrt{-g}$. The tetradic covariant derivative obeys, e.g., $\nabla_{\hat{\alpha}} V^{\hat{\beta}}=e_{\hat{\alpha}}^{\mu} e_{\nu}^{\hat{\beta}} \nabla_{\mu} V^{\nu}$. 
We observe that the reference to the local tetrad has completely disappeared from the action. For convenience, we shall work only with the global (tensorial) components $G_{\mu_{1} \cdots \mu_{\ell}}$ and $H_{\mu_{1} \cdots \mu_{\ell}}$ of the moments henceforth.

The coefficients $\mu^{(\ell)}$ and $\sigma^{(\ell)}$ entering the non-minimal action characterize the deformability and polarizability of the body under the influence of the external tidal field. They are linked to the dimensionless mass-type $k^{(\ell)}$ and current-type $j^{(\ell)}$ second Love numbers as [19]

$$
G \mu_{A}^{(\ell)}=\frac{2}{(2 \ell-1) ! !} k_{A}^{(\ell)} R_{A}^{2 \ell+1}, \quad G \sigma_{A}^{(\ell)}=\frac{\ell-1}{4(\ell+2)(2 \ell-1) ! !} j_{A}^{(\ell)} R_{A}^{2 \ell+1},
$$

where $R$ is the radius of the body (in a coordinate system such that the area of the sphere of radius $R$ is $4 \pi R^{2}$ ). In the effective description Eq. (2.7) of compact objects, only the coefficients $\mu_{A}^{(\ell)}$ and $\sigma_{A}^{(\ell)}$ are measurable. The normalization constants in the first equation (2.8) are chosen to match usual Newtonian definitions.

The polarizability coefficients (2.8) actually determine the formal PN order at which the tidal effects appear. For compact objects, indeed, the compactness parameter defined as the ratio $\mathcal{C} \sim G m /\left(R c^{2}\right)$ is of the order of one. Inserting $\mathcal{C} \sim 1$ in Eq. (2.8), we recover the fact that the dominant tidal effect is due to the mass quadrupole and is formally of the order of

$$
\epsilon_{\text {tidal }} \sim \frac{1}{c^{10}},
$$

i.e., is comparable to a $5 \mathrm{PN}$ orbital effect. With the notation (2.9) for the dominant effect, we see that the deformability coefficients in the action scale like

$$
\left\{\mu_{A}^{(\ell)}, \sigma_{A}^{(\ell)}\right\}=\mathcal{O}\left(\frac{\epsilon_{\text {tidal }}}{c^{4 \ell-8}}\right) .
$$

As we aim at computing tidal effects up to the NNL/2PN order, inspection of the action (2.7) shows that we may consider only the mass quadrupole, current quadrupole and mass octupole interactions:

$$
S_{m}=\sum_{A} \int \mathrm{d} \tau_{A}\left[-m_{A} c^{2}+\frac{\mu_{A}^{(2)}}{4} G_{\mu \nu}^{A} G_{A}^{\mu \nu}+\frac{\sigma_{A}^{(2)}}{6 c^{2}} H_{\mu \nu}^{A} H_{A}^{\mu \nu}+\frac{\mu_{A}^{(3)}}{12} G_{\lambda \mu \nu}^{A} G_{A}^{\lambda \mu \nu}+\mathcal{O}\left(\frac{\epsilon_{\text {tidal }}}{c^{6}}\right)\right],
$$

where the specified remainder means that we neglect higher order - NNNL and beyond - terms. Direct application of the general scaling relation (2.10) shows that $\mu^{(2)}=\mathcal{O}\left(\epsilon_{\text {tidal }}\right), \sigma^{(2)}=\mathcal{O}\left(\epsilon_{\text {tidal }}\right)$, and $\mu^{(3)}=\mathcal{O}\left(\epsilon_{\text {tidal }} / c^{4}\right)$. Thus, the first tidal term in (2.11) yields the leading effect together with NL and NNL corrections, the second tidal term contains NL and NNL effects (because of the explicit factor $1 / c^{2}$ in the action), whereas the third one represents a purely NNL effect.

\section{METRIC AND REQUIRED ELEMENTARY POTENTIALS}

To build an action for the sole matter variables, we (i) start from the Einstein-Hilbert action (2.1) with the nonminimal matter couplings (2.2), (ii) solve the Einstein field equations resulting from the metric variation by means of a direct PN iteration, (iii) insert the explicit PN solution for the metric back into Eqs. (2.1)-(2.2), which defines the so-called (PN) Fokker action, say $S_{\mathrm{F}}$. An important point is that, at the NNL/2PN level, it is necessary and sufficient to insert into Eqs. (2.1)-(2.2) the metric generated by a system of point particles, omitting all the terms associated with the body internal structure.

To see this, we write, as in Ref. [36], the (allegedly "exact") PN solution of the Einstein field equations in terms of the gothic metric deviation $h^{\mu \nu}=\sqrt{-g} g^{\mu \nu}-\eta^{\mu \nu}$, using the particular vector variable

$$
h=\left(h^{00 i i}, h^{0 i}, h^{i j}\right), \quad \text { with } \quad h^{00 i i} \equiv h^{00}+\delta_{i j} h^{i j} .
$$

We already know that the dominant tidal effect is due to the mass quadrupole moment and pops up in the EoM at the order (2.9). We can thus write the previous solution as

$$
h=h_{\mathrm{pp}}+h_{\text {tidal }},
$$

where the first term is just the result for the metric generated by point-particles (pp) without internal structure, and where the tidal corrections therein are at least of the order of (with obvious notation)

$$
h_{\text {tidal }}=\mathcal{O}\left(\frac{\epsilon_{\text {tidal }}}{c^{2}}, \frac{\epsilon_{\text {tidal }}}{c^{3}}, \frac{\epsilon_{\text {tidal }}}{c^{4}}\right) .
$$


Since $h$ is an exact solution of the Einstein field equations we have $\delta S_{\mathrm{F}} / \delta h=0$, which implies that the functional derivative of the Fokker action evaluated for the "approximate" solution $h_{\mathrm{pp}}$ will be of the order of the committed error, namely (taking into account the coupling constant $c^{4} /(16 \pi G)$ in the field equations)

$$
\frac{\delta S_{\mathrm{F}}}{\delta h}\left[h_{\mathrm{pp}}\right]=\mathcal{O}\left(c^{2} \epsilon_{\text {tidal }}, c \epsilon_{\text {tidal }}, \epsilon_{\text {tidal }}\right) .
$$

The two facts (3.3) and (3.4) combined together in a Taylor expansion of the action imply that

$$
\begin{aligned}
S_{\mathrm{F}}[h] & =S_{\mathrm{F}}\left[h_{\mathrm{pp}}\right]+\int \mathrm{d}^{4} x \frac{\delta S_{\mathrm{F}}}{\delta h}\left[h_{\mathrm{pp}}\right] h_{\text {tidal }}+\mathcal{O}\left(h_{\text {tidal }}^{2}\right) \\
& =S_{\mathrm{F}}\left[h_{\mathrm{pp}}\right]+\mathcal{O}\left(\epsilon_{\text {tidal }}^{2}\right)
\end{aligned}
$$

and we conclude that the final remainder $\mathcal{O}\left(\epsilon_{\text {tidal }}^{2}\right)$ is at least comparable to a $10 \mathrm{PN}$ effect $\mathcal{O}\left(c^{-20}\right)$ [see Eq. (2.9)]. Therefore, it is amply sufficient to insert into the Fokker action the metric $h_{\mathrm{pp}}$ for point particles without internal structure. We can recover this conclusion from a general statement proved in Ref. [36] and called the method " $n+2$ ", according to which, in order to control the Fokker action at some $n \mathrm{PN}$ order, it is necessary and sufficient to insert the components of the metric $h$ with all the PN corrections up to the order $1 / c^{n+2}$ included. In our case, we want the Fokker action up to the NNL order, which means formally $7 \mathrm{PN}$, hence $n=7$, so that we require the metric up to the maximal order $1 / c^{9}$ while tidal effects are of higher order [see Eq. (3.3)]. The same argument has also been shown and used in Sec. II.E of Ref. [19].

In this paper, we shall not try to compute the full action, including all the terms up to NNL order $\mathcal{O}\left(\epsilon_{\text {tidal }} / c^{4}\right)$, but only the tidal NNL contributions therein, proceeding essentially as in Ref. [19] although staying in harmonic coordinates. Consequently, we shall need the point-particle metric up to the $2 \mathrm{PN}$ order only, so as to obtain the regularized Weyl or Riemann tensor of point particles at the $2 \mathrm{PN}$ order, which is the minimum requirement to control the tidal moments at the same accuracy level:

$$
\begin{aligned}
G_{\mu \nu}^{A} & =-c^{2}\left[R_{\mu \rho \nu \sigma}\right]_{A} u_{A}^{\rho} u_{A}^{\sigma}, \\
H_{\mu \nu}^{A} & =2 c^{3}\left[R_{(\mu \underline{\rho} \nu) \sigma}^{*}\right]_{A} u_{A}^{\rho} u_{A}^{\sigma}, \\
G_{\lambda \mu \nu}^{A} & =-c^{2}\left[\nabla_{(\lambda}^{\perp} R_{\mu \underline{\rho} \nu) \sigma}\right]_{A} u_{A}^{\rho} u_{A}^{\sigma} .
\end{aligned}
$$

Remind that, for this calculation, the Weyl and the Riemann tensors give an equivalent dynamics (see Appendix B). On the other hand, one can show that replacing the STF operator by the symmetrization operator in the definitions (2.5) for the mass quadrupole, current quadrupole and mass octupole moments does not affect the values of those tensors. The resulting expressions, provided in Appendix B, are simpler than the original formulae. The tensors (3.6) are then obtained by substituting there the Riemann tensor to the Weyl one. However, the off-shell mass-type tidal moments defined in this manner are no longer trace-free, contrary to their Weyl counterparts.

At the $2 \mathrm{PN}$ order, the metric of a general matter system in harmonic coordinates can be parametrized by the set of potentials $\left\{V, V_{i}, \hat{W}_{i j}, \hat{R}_{i}, \hat{X}\right\}$ in the following way:

$$
\begin{aligned}
& g_{00}=-1+\frac{2 V}{c^{2}}-\frac{2 V^{2}}{c^{4}}+\frac{8}{c^{6}}\left(\hat{X}+V_{i} V_{i}+\frac{V^{3}}{6}\right)+\mathcal{O}\left(\frac{1}{c^{8}}\right), \\
& g_{0 i}=-\frac{4 V_{i}}{c^{3}}-\frac{8 \hat{R}_{i}}{c^{5}}+\mathcal{O}\left(\frac{1}{c^{7}}\right), \\
& g_{i j}=\delta_{i j}\left(1+\frac{2 V}{c^{2}}+\frac{2 V^{2}}{c^{4}}\right)+\frac{4 \hat{W}_{i j}}{c^{4}}+\mathcal{O}\left(\frac{1}{c^{6}}\right) .
\end{aligned}
$$

These potentials admit a non-zero finite Newtonian limit and solve the flat-space wave equations (with $\square=\eta^{\mu \nu} \partial_{\mu \nu}^{2}$ )

$$
\begin{aligned}
\square V & =-4 \pi G \sigma \\
\square V_{i} & =-4 \pi G \sigma_{i} \\
\square \hat{W}_{i j} & =-4 \pi G\left(\sigma_{i j}-\delta_{i j} \sigma_{k k}\right)-\partial_{i} V \partial_{j} V \\
\square \hat{R}_{i} & =-4 \pi G\left(V \sigma_{i}-V_{i} \sigma\right)-2 \partial_{k} V \partial_{i} V_{k}-\frac{3}{2} \partial_{t} V \partial_{i} V \\
\square \hat{X} & =-4 \pi G V \sigma_{k k}+2 V_{k} \partial_{t} \partial_{k} V+V \partial_{t}^{2} V+\frac{3}{2}\left(\partial_{t} V\right)^{2}-2 \partial_{i} V_{j} \partial_{j} V_{i}+\hat{W}_{i j} \partial_{i j} V,
\end{aligned}
$$


where the matter source densities are defined in terms of the components of the matter stress-energy tensor as

$$
\sigma=\frac{T^{00}+T^{i i}}{c^{2}}, \quad \sigma_{i}=\frac{T^{0 i}}{c}, \quad \sigma_{i j}=T^{i j},
$$

with $T^{i i}=\delta_{i j} T^{i j}$. To perform a consistent Fokker reduction of the original action, the solutions of Eqs. (3.8) must be in principle constructed with the symmetric Green function, which kills all contributions of odd powers of $1 / c$ at the current approximation level. As discussed above, thanks to the properties of the Fokker action, we only need the metric produced by point-like particles and can neglect tidal effects when inserting the metric (3.7) into the Fokker action. Therefore, we shall just compute the potentials for point particles without including any internal structure effect. The requested potentials have already been published elsewhere [37], except that we compute here their off-shell values, without replacement of accelerations by means of the EoM (we then call them the "unreduced" potentials). However, it is known that the replacement of accelerations in the action is equivalent to performing an unphysical shift of the particles' worldlines [38]. We have checked that, indeed, by inserting the reduced ("on-shell") versions of the potentials into the action, the final gauge invariant result for the conserved energy reduced to circular orbits, which we shall obtain below [in Eq. (6.5)], comes out the same.

For point particles without spins the matter source terms (3.9) take the form

$$
\begin{aligned}
\sigma(\mathbf{x}, t) & =\sum_{A} \tilde{\mu}_{A}(t) \delta^{(3)}\left(\mathbf{x}-\boldsymbol{y}_{A}(t)\right), \\
\sigma_{i}(\mathbf{x}, t) & =\sum_{A} \mu_{A} v_{A}^{i} \delta^{(3)}\left(\mathbf{x}-\boldsymbol{y}_{A}(t)\right), \\
\sigma_{i j}(\mathbf{x}, t) & =\sum_{A} \mu_{A} v_{A}^{i} v_{A}^{j} \delta^{(3)}\left(\mathbf{x}-\boldsymbol{y}_{A}(t)\right),
\end{aligned}
$$

where the three-dimensional Dirac function is confined to the worldline $\boldsymbol{y}_{A}(t)$ and we pose for the effective time-varying masses (with $m_{A}$ the constant PN mass)

$$
\mu_{A}(t)=\frac{m_{A} c}{\sqrt{\left[g g_{\mu \nu}\right]_{A} v_{A}^{\mu} v_{A}^{\nu}}}, \quad \tilde{\mu}_{A}=\left(1+\frac{\boldsymbol{v}_{A}^{2}}{c^{2}}\right) \mu_{A},
$$

In Eqs. (3.10)-(3.11), the worldlines are parametrized by the coordinate time $t=x^{0} / c$ of the harmonic coordinates; the coordinate velocities are $v_{A}^{\mu}=\left(c, v_{A}^{i}\right)$, with $v_{A}^{i}=c u_{A}^{i} / u_{A}^{0}=\mathrm{d} y_{A}^{i} / \mathrm{d} t$, and the relativistic Lorentz factor reads $u_{A}^{0}=\left(-\left[g_{\mu \nu}\right]_{A} v_{A}^{\mu} v_{A}^{\nu} / c^{2}\right)^{-1 / 2}$. The metric is computed at the location of the particle $A$ following dimensional regularization; in particular, we have $\left[g g_{\mu \nu}\right]_{A}=[g]_{A}\left[g_{\mu \nu}\right]_{A}$ in (3.11). As we said, in practical calculations, we use the Hadamard regularization, which is equivalent to dimensional regularization up to the relatively low NNL/2PN order $[19,34]$.

To summarize, the metric in Eq. (3.7) is not the full metric and does not contain tidal effects. However, as proved in Eqs. (3.1) to (3.5), it is sufficient to insert into the Fokker action $S_{g}+S_{m}$ needed to obtain the tidal effects in the equations of motion at the requested order. In our work, the full metric is not controlled. Indeed, we only compute the equations of motion of the particles (and the associated conserved quantities). With the Fokker method, we do not need nor have access to the full metric outside the particle's worldlines.

\section{TIDAL EFFECTS IN THE EQUATIONS OF MOTION TO NNL ORDER}

From the discussion in the previous section, we know that, up to the NNL order, the only terms in the Fokker action that depend on the bodies' internal structure are those that are explicitly present into the matter action (2.11). Here, we provide the results for the (coordinate basis components of the) tidal mass-quadrupole, mass-octupole and current-quadrupole moments at the NNL order felt by the body 1, i.e., regularized at the point 1 . We find ${ }^{6}$

$$
\left[G_{i j}\right]_{1}=\frac{G m_{2}}{r_{12}^{3}}\left[3 n_{12\langle i} n_{12 j\rangle}+\frac{1}{c^{2}}\left[n_{12\langle i} n_{12 j\rangle}\left(-\frac{15}{2}\left(n_{12} v_{2}\right)^{2}+6 v_{12}^{2}-\frac{3}{2} r_{12}\left(n_{12} a_{2}\right)-\frac{3 G m_{1}}{r_{12}}-\frac{3 G m_{2}}{r_{12}}\right)\right.\right.
$$

\footnotetext{
${ }^{6}$ The notation $r_{12}=\left|\boldsymbol{y}_{1}-\boldsymbol{y}_{2}\right|$ represents the Euclidean distance between the two bodies (at constant time $y_{1}^{0}=y_{2}^{0}=c t$ ); the unit direction from body 2 to body 1 is then $n_{12}^{i}=\left(y_{1}^{i}-y_{2}^{i}\right) / r_{12} ; v_{12}^{i}=v_{1}^{i}-v_{2}^{i}$ stands for the relative velocity; the usual Euclidean scalar product of vectors is denoted with parentheses, e.g. $\left(n_{12} v_{1}\right)=\boldsymbol{n}_{12} \cdot \boldsymbol{v}_{1}$; the cross product is denoted, e.g. $\left(n_{12} \times v_{12}\right)_{i}$, and the mixed product, e.g. $\left(n_{12}, v_{1}, v_{2}\right)=\left(n_{12} v_{1} \times v_{2}\right)$. All calculations are done with the software Mathematica and the tensor package $x A c t$ [39].
} 


$$
\begin{aligned}
& -6 n_{12\langle i} v_{1 j\rangle}\left(n_{12} v_{12}\right)+2 v_{1\langle i} v_{1 j\rangle}+n_{12\langle i} v_{2 j\rangle}\left(12\left(n_{12} v_{1}\right)-6\left(n_{12} v_{2}\right)\right)-6 v_{1\langle i} v_{2 j\rangle}+3 v_{2\langle i} v_{2 j\rangle}-3 a_{2\langle i} n_{12 j\rangle} r_{12} \\
& \left.+\delta_{i j}\left(\left(n_{12} v_{1}\right)^{2}-\frac{1}{3} v_{1}^{2}\right)\right]+\frac{1}{c^{4}}\left\{n _ { 1 2 \langle i } n _ { 1 2 j \rangle } \left[\frac{105}{8}\left(n_{12} v_{2}\right)^{4}+30\left(n_{12} v_{2}\right)^{2}\left(v_{1} v_{2}\right)+6\left(v_{1} v_{2}\right)^{2}-15\left(n_{12} v_{2}\right)^{2} v_{1}^{2}\right.\right. \\
& -12\left(v_{1} v_{2}\right) v_{1}^{2}+6 v_{1}^{4}-\frac{45}{2}\left(n_{12} v_{2}\right)^{2} v_{2}^{2}-12\left(v_{1} v_{2}\right) v_{2}^{2}+6 v_{1}^{2} v_{2}^{2}+6 v_{2}^{4}+G m_{2}\left(n_{12} a_{2}\right) \\
& +\frac{G m_{1}}{r_{12}}\left(-\frac{291}{2}\left(n_{12} v_{1}\right)^{2}+291\left(n_{12} v_{1}\right)\left(n_{12} v_{2}\right)-\frac{273}{2}\left(n_{12} v_{2}\right)^{2}+35 v_{12}^{2}\right)+G m_{1}\left(14\left(n_{12} a_{1}\right)-10\left(n_{12} a_{2}\right)\right) \\
& +\frac{G m_{2}}{r_{12}}\left(9\left(n_{12} v_{2}\right)^{2}+18 v_{12}^{2}\right)+\frac{1}{8} r_{12}^{3}\left(\ddot{a}_{2} n_{12}\right)-\frac{15 G^{2} m_{1}^{2}}{14 r_{12}^{2}}+\frac{35 G^{2} m_{1} m_{2}}{r_{12}^{2}}+\frac{5 G^{2} m_{2}^{2}}{r_{12}^{2}}+r_{12}\left(12\left(v_{1} a_{2}\right)\left(n_{12} v_{2}\right)\right. \\
& \left.-\frac{27}{2}\left(v_{2} a_{2}\right)\left(n_{12} v_{2}\right)+\frac{45}{4}\left(n_{12} a_{2}\right)\left(n_{12} v_{2}\right)^{2}+6\left(n_{12} a_{2}\right)\left(v_{1} v_{2}\right)-3\left(n_{12} a_{2}\right) v_{1}^{2}-\frac{9}{2}\left(n_{12} a_{2}\right) v_{2}^{2}\right) \\
& \left.+r_{12}^{2}\left(\frac{9}{8}\left(n_{12} a_{2}\right)^{2}-\frac{15}{8} a_{2}^{2}+\frac{3}{2}\left(n_{12} v_{2}\right)\left(n_{12} \dot{a}_{2}\right)+2\left(v_{1} \dot{a}_{2}\right)-2\left(v_{2} \dot{a}_{2}\right)\right)\right]+n_{12\langle i} v_{1 j\rangle}\left[\frac{62 G m_{1}}{r_{12}}\left(n_{12} v_{12}\right)\right. \\
& -\frac{18 G m_{2}}{r_{12}}\left(n_{12} v_{12}\right)+15\left(n_{12} v_{1}\right)\left(n_{12} v_{2}\right)^{2}-15\left(n_{12} v_{2}\right)^{3}+6\left(n_{12} v_{2}\right)\left(v_{1} v_{2}\right)+6\left(n_{12} v_{2}\right) v_{12}{ }^{2}-6\left(n_{12} v_{1}\right) v_{1}^{2} \\
& \left.+r_{12}\left(-\left(v_{12} a_{2}\right)+3\left(n_{12} a_{2}\right)\left(n_{12} v_{1}\right)-9\left(n_{12} a_{2}\right)\left(n_{12} v_{2}\right)\right)-r_{12}^{2}\left(n_{12} \dot{a}_{2}\right)\right]+v_{1\langle i} v_{1 j\rangle}\left(-3\left(n_{12} v_{2}\right)^{2}+2 v_{1}^{2}\right. \\
& \left.-r_{12}\left(n_{12} a_{2}\right)-\frac{3 G m_{1}}{r_{12}}+\frac{6 G m_{2}}{r_{12}}\right)+n_{12\langle i} v_{2 j\rangle}\left[-30\left(n_{12} v_{1}\right)\left(n_{12} v_{2}\right)^{2}+15\left(n_{12} v_{2}\right)^{3}-12\left(n_{12} v_{1}\right)\left(v_{1} v_{2}\right)\right. \\
& +12\left(n_{12} v_{1}\right) v_{1}^{2}+12\left(n_{12} v_{1}\right) v_{2}^{2}-6\left(n_{12} v_{2}\right) v_{2}^{2}+\frac{G m_{1}}{r_{12}}\left(-68\left(n_{12} v_{1}\right)+62\left(n_{12} v_{2}\right)\right)+\frac{G m_{2}}{r_{12}}\left(12\left(n_{12} v_{1}\right)\right. \\
& \left.\left.-18\left(n_{12} v_{2}\right)\right)+r_{12}^{2}\left(n_{12} \dot{a}_{2}\right)+r_{12}\left(-2\left(v_{1} a_{2}\right)-6\left(n_{12} a_{2}\right)\left(n_{12} v_{1}\right)-\left(v_{2} a_{2}\right)+9\left(n_{12} a_{2}\right)\left(n_{12} v_{2}\right)\right)\right] \\
& +v_{1\langle i} v_{2 j\rangle}\left(-6\left(n_{12} v_{1}\right)\left(n_{12} v_{2}\right)+15\left(n_{12} v_{2}\right)^{2}-6\left(v_{1} v_{2}\right)-6 v_{12}^{2}+5 r_{12}\left(n_{12} a_{2}\right)+\frac{8 G m_{1}}{r_{12}}-\frac{10 G m_{2}}{r_{12}}\right) \\
& +v_{2\langle i} v_{2 j\rangle}\left(6\left(n_{12} v_{1}\right)^{2}-\frac{15}{2}\left(n_{12} v_{2}\right)^{2}+3 v_{2}^{2}-\frac{5}{2} r_{12}\left(n_{12} a_{2}\right)-\frac{4 G m_{1}}{r_{12}}+\frac{5 G m_{2}}{r_{12}}\right)+4 G m_{1} a_{1\langle i} n_{12 j\rangle} \\
& +a_{2\langle i} n_{12 j\rangle}\left[r_{12}\left(-12\left(n_{12} v_{1}\right)\left(n_{12} v_{2}\right)+\frac{27}{2}\left(n_{12} v_{2}\right)^{2}+4\left(v_{1} v_{2}\right)-2 v_{1}^{2}-5 v_{2}^{2}\right)+\frac{9}{2} r_{12}^{2}\left(n_{12} a_{2}\right)-3 G m_{1}\right. \\
& \left.-G m_{2}\right]+a_{2\langle i} v_{1 j\rangle} r_{12}\left(-\left(n_{12} v_{1}\right)+7\left(n_{12} v_{2}\right)\right)+a_{2\langle i} v_{2 j\rangle} r_{12}\left(-2\left(n_{12} v_{1}\right)-7\left(n_{12} v_{2}\right)\right)-\frac{5}{4} a_{2\langle i} a_{2 j\rangle} r_{12}^{2} \\
& +n_{12\langle i} \dot{a}_{2 j\rangle} r_{12}^{2}\left(-2\left(n_{12} v_{1}\right)+5\left(n_{12} v_{2}\right)\right)+3 v_{1\langle i} \dot{a}_{2 j\rangle} r_{12}^{2}-3 v_{2\langle i} \dot{a}_{2 j\rangle} r_{12}^{2}+\frac{7}{4} n_{12\langle i} \ddot{a}_{2 j\rangle} r_{12}^{3}+\delta_{i j}\left[-\frac{5}{2}\left(n_{12} v_{1}\right)^{2}\left(n_{12} v_{2}\right)^{2}\right. \\
& -2\left(n_{12} v_{1}\right)\left(n_{12} v_{2}\right)\left(v_{1} v_{2}\right)+\left(v_{1} v_{2}\right)^{2}+\left(n_{12} v_{1}\right)^{2} v_{1}^{2}+\frac{3}{2}\left(n_{12} v_{2}\right)^{2} v_{1}^{2}-\frac{1}{3} v_{1}^{4}+2\left(n_{12} v_{1}\right)^{2} v_{2}^{2}-v_{1}^{2} v_{2}^{2} \\
& -\frac{4}{3} G m_{1}\left(n_{12} a_{2}\right)+\frac{G m_{1}}{r_{12}}\left(-\frac{16}{3}\left(n_{12} v_{12}\right)^{2}-\left(n_{12} v_{1}\right)^{2}+\frac{4}{3} v_{12}^{2}+\frac{1}{3} v_{1}^{2}\right)+\frac{G m_{2}}{r_{12}}\left(4\left(n_{12} v_{12}\right)^{2}-\left(n_{12} v_{1}\right)^{2}\right. \\
& \left.-\frac{4}{3} v_{12}^{2}+\frac{1}{3} v_{1}^{2}\right)+r_{12}\left(\frac{4}{3}\left(v_{12} a_{2}\right)\left(n_{12} v_{12}\right)-\left(v_{1} a_{2}\right)\left(n_{12} v_{1}\right)-\frac{1}{2}\left(n_{12} a_{2}\right)\left(n_{12} v_{1}\right)^{2}+\frac{1}{2}\left(n_{12} a_{2}\right) v_{1}^{2}\right) \\
& \left.\left.\left.-\frac{16 G^{2} m_{1} m_{2}}{3 r_{12}^{2}}+\frac{2 G^{2} m_{2}^{2}}{3 r_{12}^{2}}+r_{12}^{2}\left(\frac{4}{3} a_{2}^{2}-\frac{4}{3}\left(v_{1} \dot{a}_{2}\right)+\frac{4}{3}\left(v_{2} \dot{a}_{2}\right)\right)\right]\right\}\right]+\mathcal{O}\left(\frac{1}{c^{6}}\right) \\
& {\left[H_{i j}\right]_{1}=\frac{G m_{2}}{r_{12}^{3}}\left\{12\left(n_{12} \times v_{12}\right)_{\langle i} n_{12 j\rangle}+\frac{1}{c^{2}}\left[( n _ { 1 2 } \times v _ { 1 2 } ) _ { \langle i } n _ { 1 2 j \rangle } \left(-30\left(n_{12} v_{2}\right)^{2}+12\left(v_{1} v_{2}\right)+12 v_{12}{ }^{2}-6 r_{12}\left(n_{12} a_{2}\right)\right.\right.\right.} \\
& \left.+\frac{4 G m_{1}}{r_{12}}+\frac{12 G m_{2}}{r_{12}}\right)-12\left(a_{2} \times n_{12}\right)_{\langle i} n_{12 j\rangle} r_{12}\left(n_{12} v_{2}\right)+12\left(n_{12} \times v_{12}\right)_{\langle i} v_{2 j\rangle}\left(n_{12} v_{1}\right)-2\left(a_{2} \times v_{12}\right)_{\langle i} n_{12 j\rangle} r_{12} \\
& \left.\left.-2 a_{2\langle i}\left(n_{12} \times v_{12}\right)_{j\rangle} r_{12}+2\left(n_{12} \times \dot{a}_{2}\right)_{\langle i} n_{12 j\rangle} r_{12}^{2}+4 \delta_{i j}\left(n_{12}, v_{1}, v_{2}\right)\left(n_{12} v_{1}\right)\right]\right\}+\mathcal{O}\left(\frac{1}{c^{4}}\right), \\
& {\left[G_{i j k}\right]_{1}=-\frac{15 G m_{2} n_{12\langle i} n_{12 j} n_{12 k\rangle}}{r_{12}^{4}}+\mathcal{O}\left(\frac{1}{c^{2}}\right)}
\end{aligned}
$$


The other components of the tidal moments are readily obtained from, e.g., the relations $\left[G_{0 i}\right]_{1}=-v_{1}^{j}\left[G_{i j}\right]_{1} / c$ and $\left[G_{00}\right]_{1}=v_{1}^{i} v_{1}^{j}\left[G_{i j}\right]_{1} / c^{2}$, which are equivalent to $\left[G_{\hat{0} \hat{0}}\right]_{1}=\left[G_{\hat{0} \hat{i}}\right]_{1}=0$ in tetradic notation. In Eqs. (4.1), most of the terms are STF, which we denote by angular brackets surounding the indices. Note however, as mentioned in Sec. III, the appearance of pure trace contributions, due to the fact that we have not resorted here to tetradic projections and have used the Riemann tensor instead of the Weyl tensor [see the discussion in Appendix B].

With the latter results and the 2PN metric (3.7) in hands, it is straightforward to get the Lagrangian up to the relative NNL/2PN order for the finite-size tidal contributions. As usual, we apply a number of procedures to eliminate multiple time derivatives of the accelerations and reduce the numbers of terms, in particular removing those that contain higher time derivatives of the accelerations by adding suitable double-zero terms and total time derivatives [40]. Recalling our notation introduced in Eq. (3.2), we write

$$
L=L_{\mathrm{pp}}+L_{\text {tidal }}
$$

where, to be consistent with the NNL order truncation, we recall here the Lagrangian for point particles up to $2 \mathrm{PN}$ order in harmonic coordinates, which is a generalized Lagrangian depending on positions $y_{A}^{i}(t)$, velocities $v_{A}^{i}(t)$, as well as accelerations $a_{A}^{i}(t)=\mathrm{d} v_{A}^{i} / \mathrm{d} t$ (see, e.g., Eq. (209) of [41]):

$$
\begin{aligned}
L_{\mathrm{pp}}= & \frac{m_{1} v_{1}^{2}}{2}+\frac{G m_{1} m_{2}}{2 r_{12}} \\
+ & \frac{1}{c^{2}}\left\{-\frac{G^{2} m_{1}^{2} m_{2}}{2 r_{12}^{2}}+\frac{m_{1} v_{1}^{4}}{8}+\frac{G m_{1} m_{2}}{r_{12}}\left(-\frac{1}{4}\left(n_{12} v_{1}\right)\left(n_{12} v_{2}\right)+\frac{3}{2} v_{1}^{2}-\frac{7}{4}\left(v_{1} v_{2}\right)\right)\right\} \\
+ & \frac{1}{c^{4}}\left\{\frac{G^{3} m_{1}^{3} m_{2}}{2 r_{12}^{3}}+\frac{19 G^{3} m_{1}^{2} m_{2}^{2}}{8 r_{12}^{3}}\right. \\
& +\frac{G^{2} m_{1}^{2} m_{2}}{r_{12}^{2}}\left(\frac{7}{2}\left(n_{12} v_{1}\right)^{2}-\frac{7}{2}\left(n_{12} v_{1}\right)\left(n_{12} v_{2}\right)+\frac{1}{2}\left(n_{12} v_{2}\right)^{2}+\frac{1}{4} v_{1}^{2}-\frac{7}{4}\left(v_{1} v_{2}\right)+\frac{7}{4} v_{2}^{2}\right) \\
& +\frac{G m_{1} m_{2}}{r_{12}}\left(\frac{3}{16}\left(n_{12} v_{1}\right)^{2}\left(n_{12} v_{2}\right)^{2}-\frac{7}{8}\left(n_{12} v_{2}\right)^{2} v_{1}^{2}+\frac{7}{8} v_{1}^{4}+\frac{3}{4}\left(n_{12} v_{1}\right)\left(n_{12} v_{2}\right)\left(v_{1} v_{2}\right)\right. \\
& \left.\quad-2 v_{1}^{2}\left(v_{1} v_{2}\right)+\frac{1}{8}\left(v_{1} v_{2}\right)^{2}+\frac{15}{16} v_{1}^{2} v_{2}^{2}\right)+\frac{m_{1} v_{1}^{6}}{16} \\
& \left.+G m_{1} m_{2}\left(-\frac{7}{4}\left(a_{1} v_{2}\right)\left(n_{12} v_{2}\right)-\frac{1}{8}\left(n_{12} a_{1}\right)\left(n_{12} v_{2}\right)^{2}+\frac{7}{8}\left(n_{12} a_{1}\right) v_{2}^{2}\right)\right\}+1 \leftrightarrow 2+\mathcal{O}\left(\frac{1}{c^{5}}\right) .
\end{aligned}
$$

To the terms given above, we must add their symmetric counterpart in the exchange of the two particles, as indicated by the notation $1 \leftrightarrow 2$. Now, the main result of the present paper is the complete expression of the tidal part of the Lagrangian up to the $\mathrm{NNL} / 2 \mathrm{PN}$ order in harmonic coordinates. It reads

$$
\begin{aligned}
L_{\text {tidal }} & =\frac{G^{2} m_{2}^{2}}{r_{12}^{6}}\left\{\frac{3}{2} \mu_{1}^{(2)}+\frac{1}{c^{2}}\left[\mu_{1}^{(2)}\left(-\frac{9}{2}\left(n_{12} v_{1}\right)^{2}-18\left(n_{12} v_{1}\right)\left(n_{12} v_{2}\right)+18\left(n_{12} v_{2}\right)^{2}-\frac{9}{2}\left(v_{1} v_{2}\right)+\frac{15}{4} v_{1}^{2}\right)\right.\right. \\
& \left.+\sigma_{1}^{(2)}\left(-12\left(n_{12} v_{12}\right)^{2}+12 v_{12}^{2}\right)-\frac{3 G m_{1} \mu_{1}^{(2)}}{r_{12}}-\frac{21 G m_{2} \mu_{1}^{(2)}}{2 r_{12}}\right]+\frac{1}{c^{4}}\left[\mu _ { 1 } ^ { ( 2 ) } \left(\frac{9}{2}\left(n_{12} v_{1}\right)^{4}\right.\right. \\
& -18\left(n_{12} v_{1}\right)^{3}\left(n_{12} v_{2}\right)+45\left(n_{12} v_{1}\right)^{2}\left(n_{12} v_{2}\right)^{2}-54\left(n_{12} v_{1}\right)\left(n_{12} v_{2}\right)^{3}+\frac{63}{2}\left(n_{12} v_{2}\right)^{4}+9\left(n_{12} v_{1}\right)\left(n_{12} v_{2}\right)\left(v_{1} v_{2}\right) \\
& -18\left(n_{12} v_{2}\right)^{2}\left(v_{1} v_{2}\right)+\frac{9}{2}\left(v_{1} v_{2}\right)^{2}-9\left(n_{12} v_{1}\right)^{2} v_{12}^{2}+27\left(n_{12} v_{1}\right)\left(n_{12} v_{2}\right) v_{12}^{2}-36\left(n_{12} v_{2}\right)^{2} v_{12}^{2} \\
& +9\left(v_{1} v_{2}\right) v_{12}^{2}+9 v_{12}^{4}-\frac{9}{4}\left(n_{12} v_{1}\right)^{2} v_{1}^{2}-\frac{9}{2}\left(n_{12} v_{1}\right)\left(n_{12} v_{2}\right) v_{1}^{2}+\frac{27}{2}\left(n_{12} v_{2}\right)^{2} v_{1}^{2}-9\left(v_{1} v_{2}\right) v_{1}^{2} \\
& \left.-\frac{27}{4} v_{12}^{2} v_{1}^{2}+\frac{69}{16} v_{1}^{4}\right)+\mu_{1}^{(2)} r_{12}\left(-12\left(v_{12} a_{2}\right)\left(n_{12} v_{1}\right)+60\left(n_{12} a_{2}\right)\left(n_{12} v_{1}\right)^{2}+21\left(v_{12} a_{2}\right)\left(n_{12} v_{2}\right)\right. \\
& -\frac{9}{2}\left(v_{1} a_{2}\right)\left(n_{12} v_{2}\right)-102\left(n_{12} a_{2}\right)\left(n_{12} v_{1}\right)\left(n_{12} v_{2}\right)+60\left(n_{12} a_{2}\right)\left(n_{12} v_{2}\right)^{2}+\frac{69}{2}\left(n_{12} a_{2}\right)\left(v_{1} v_{2}\right)-\frac{69}{4}\left(n_{12} a_{2}\right) v_{1}^{2} \\
& \left.-\frac{39}{2}\left(n_{12} a_{2}\right) v_{2}^{2}\right)+\sigma_{1}^{(2)}\left(60\left(n_{12} v_{12}\right)^{4}-96\left(n_{12} v_{12}\right)^{3}\left(n_{12} v_{1}\right)+48\left(n_{12} v_{12}\right)^{2}\left(n_{12} v_{1}\right)^{2}-24\left(n_{12} v_{12}\right)^{2}\left(v_{1} v_{2}\right)\right. \\
& +24\left(n_{12} v_{12}\right)\left(n_{12} v_{1}\right)\left(v_{1} v_{2}\right)+12\left(v_{1} v_{2}\right)^{2}-84\left(n_{12} v_{12}\right)^{2} v_{12}^{2}+96\left(n_{12} v_{12}\right)\left(n_{12} v_{1}\right) v_{12}^{2}-36\left(n_{12} v_{1}\right)^{2} v_{12}^{2}
\end{aligned}
$$




$$
\begin{aligned}
& \left.+24\left(v_{1} v_{2}\right) v_{12}^{2}+24 v_{12}^{4}+18\left(n_{12} v_{12}\right)^{2} v_{1}^{2}-24\left(n_{12} v_{12}\right)\left(n_{12} v_{1}\right) v_{1}^{2}-24\left(v_{1} v_{2}\right) v_{1}^{2}-18 v_{12}^{2} v_{1}^{2}+12 v_{1}^{4}\right) \\
& +\sigma_{1}^{(2)} r_{12}\left(16\left(n_{12} a_{2}\right)\left(n_{12} v_{12}\right)^{2}+24\left(v_{12} a_{2}\right)\left(n_{12} v_{1}\right)-24\left(n_{12} a_{2}\right)\left(n_{12} v_{12}\right)\left(n_{12} v_{1}\right)-16\left(n_{12} a_{2}\right) v_{12}^{2}\right) \\
& +\frac{G m_{1} \mu_{1}^{(2)}}{r_{12}}\left(\frac{807}{8}\left(n_{12} v_{1}\right)^{2}+\frac{381}{8}\left(n_{12} v_{1}\right)\left(n_{12} v_{2}\right)-138\left(n_{12} v_{2}\right)^{2}-\frac{387}{8}\left(v_{1} v_{2}\right)+\frac{63}{8} v_{1}^{2}+42 v_{2}^{2}\right) \\
& +\frac{G m_{2} \mu_{1}^{(2)}}{r_{12}}\left(\frac{27}{2}\left(n_{12} v_{1}\right)^{2}+\frac{1051}{8}\left(n_{12} v_{1}\right)\left(n_{12} v_{2}\right)-\frac{865}{8}\left(n_{12} v_{2}\right)^{2}+\frac{83}{8}\left(v_{1} v_{2}\right)-\frac{45}{4} v_{1}^{2}+\frac{49}{8} v_{2}^{2}\right) \\
& +\frac{G m_{1} \sigma_{1}^{(2)}}{r_{12}}\left(-8\left(n_{12} v_{12}\right)^{2}+8 v_{12}^{2}\right)+\frac{G m_{2} \sigma_{1}^{(2)}}{r_{12}}\left(36\left(n_{12} v_{12}\right)^{2}-36 v_{12}^{2}\right)-\frac{60 G^{2} m_{1}^{2} \mu_{1}^{(2)}}{7 r_{12}^{2}} \\
& \left.\left.+\frac{707 G^{2} m_{1} m_{2} \mu_{1}^{(2)}}{8 r_{12}^{2}}+\frac{165 G^{2} m_{2}^{2} \mu_{1}^{(2)}}{4 r_{12}^{2}}\right]+\frac{15 \mu_{1}^{(3)}}{2 r_{12}^{2}}\right\}+1 \leftrightarrow 2+\mathcal{O}\left(\frac{\epsilon_{\text {tidal }}}{c^{6}}\right) .
\end{aligned}
$$

Note that the last term, although it does not contain any explicit $1 / c$-factor, is actually a NNL term [see Eq. (2.10)].

The long EoM derived by varying the Lagrangian (4.4) are relagated to Appendix C. We have verified that the latter EoM in harmonic coordinates stay manifestly invariant when we perform a global (PN-expanded) Lorentz boost with constant velocity $\boldsymbol{V}$. All the formulas employed to check the Lorentz invariance are given by Eqs. (3.20)-(3.23) of Ref. [42]. Furthermore, as a confirmation of the boost invariance of the EoM, we can compute the Noetherian invariant associated with this symmetry, which is nothing but the (mass weighted) position of the center of mass $G^{i}$ of the binary system. We obtain $G^{i}=G_{\mathrm{pp}}^{i}+G_{\text {tidal }}^{i}$, where the point-particle piece is given by Eq. (4.4) in [43], i.e. at the $1 \mathrm{PN}$ order by

$$
G_{\mathrm{pp}}^{i}=m_{1} y_{1}^{i}+\frac{m_{1}}{2 c^{2}}\left(v_{1}^{2}-\frac{G m_{2}}{r_{12}}\right) y_{1}^{i}+1 \leftrightarrow 2+\mathcal{O}\left(\frac{1}{c^{4}}\right)
$$

and where the dominant tidal piece appears only at the NL/1PN order and is given by

$$
G_{\text {tidal }}^{i}=\frac{3 G^{2} m_{2}^{2}}{2 r_{12}^{5} c^{2}} \mu_{1}^{(2)}\left(3 n_{12}^{i}-\frac{y_{1}^{i}}{r_{12}}\right)+1 \leftrightarrow 2+\mathcal{O}\left(\frac{\epsilon_{\text {tidal }}}{c^{4}}\right)
$$

For simplicity, since it is not needed in the following, we do not present the complicated NNL/2PN contributions beyond the result (4.6).

\section{TIDAL EFFECTS IN THE CENTER-OF-MASS FRAME}

The center-of-mass ( $\mathrm{CoM})$ frame is defined as the frame for which the equation $G^{i}=0$ holds, consistently including the tidal terms. The structure of the leading order of the EoM and energy allows one to compute the corresponding CoM quantities at the $2 \mathrm{PN}$ relative order without requesting $G^{i}$ itself at that order. By contrast, it is sufficient to know $G^{i}$ at the $1 \mathrm{PN}$ relative order for this calculation, which means including the tidal effects at the NL/1PN order as given by Eq. (4.6). Solving for $G^{i}=0$ then yields the CoM position of the particle 1 as a function of the relative separation and velocity. ${ }^{7}$ We find $y_{1}^{i}=\left(y_{1}^{i}\right)_{\mathrm{pp}}+\left(y_{1}^{i}\right)_{\text {tidal }}$, where the known 1PN expression for the point-particle piece reads

$$
\left(y_{1}^{i}\right)_{\mathrm{pp}}=\left[X_{2}+\frac{\nu \Delta}{2 c^{2}}\left(v^{2}-\frac{G m}{r}\right)\right] x^{i}+\mathcal{O}\left(\frac{1}{c^{4}}\right),
$$

with the position of the particle 2 obtained by the exchange $1 \leftrightarrow 2$. Now, the point is that, because of the tidal contribution to the CoM position found in (4.6), there also exists a NL/1PN contribution given by

$$
\left(y_{1}^{i}\right)_{\text {tidal }}=-\frac{3 G^{2} m \nu}{2 r^{6} c^{2}}\left(\Delta \mu_{+}^{(2)}+5 \mu_{-}^{(2)}\right) x^{i}+\mathcal{O}\left(\frac{\epsilon_{\text {tidal }}}{c^{4}}\right) .
$$

\footnotetext{
${ }^{7}$ We pose $x^{i}=y_{1}^{i}-y_{2}^{i}$ and $v^{i}=\mathrm{d} x^{i} / \mathrm{d} t ; r=|\boldsymbol{x}|=r_{12}$ denotes the separation, $n^{i}=x^{i} / r$ the unit direction, and we have $\dot{r}=(n v)=\boldsymbol{n} \cdot \boldsymbol{v}$; mass parameters are: the total mass $m=m_{1}+m_{2}$, the symmetric mass ratio $\nu=m_{1} m_{2} / m^{2}=X_{1} X_{2}$ and the mass difference $\Delta=X_{1}-X_{2}$, with $X_{A}=m_{A} / m$.
} 
The velocities $v_{1}^{i}=\left(v_{1}^{i}\right)_{\mathrm{pp}}+\left(v_{1}^{i}\right)_{\text {tidal }}$ are found by iteratively differentiating Eqs. (5.1)-(5.2), using in that process the full EoM, which include the tidal effect. Here and below, we define the following convenient combinations of the tidal polarizabilities:

$$
\mu_{ \pm}^{(\ell)}=\frac{1}{2}\left(\frac{m_{2}}{m_{1}} \mu_{1}^{(\ell)} \pm \frac{m_{1}}{m_{2}} \mu_{2}^{(\ell)}\right), \quad \sigma_{ \pm}^{(\ell)}=\frac{1}{2}\left(\frac{m_{2}}{m_{1}} \sigma_{1}^{(\ell)} \pm \frac{m_{1}}{m_{2}} \sigma_{2}^{(\ell)}\right)
$$

where the chosen normalisation is such that $\mu_{+}^{(\ell)}=\mu_{1}^{(\ell)}=\mu_{2}^{(\ell)}$ and $\mu_{-}^{(\ell)}=0$ when the two bodies are identical, with the same mass and internal structure. Likewise for $\sigma_{ \pm}^{(\ell)}$.

At this stage, the EoM in the CoM frame can be derived in two possible ways: either by computing the CoM acceleration $a^{i}=a_{1}^{i}-a_{2}^{i}$ directly, based on the replacement rules (5.1)-(5.2), or by getting first the expression of the Lagrangian in the CoM frame from the Lagrangian in a general frame, varying it then to recover the EoM. We resorted to the two methods and the results are in full agreement (see also [44] for further details on the second method). The CoM Lagrangian may be decomposed as $L=L_{\mathrm{pp}}+L_{\mathrm{tidal}}$, where $L_{\mathrm{pp}}$ is e.g. given by Eq. (4.2) in [45] while the tidal part is, up to NNL order

$$
\begin{aligned}
\frac{L_{\text {tidal }}}{\mu} & =\frac{G^{2} m}{r^{6}}\left\{3 \mu_{+}^{(2)}+\frac{1}{c^{2}}\left\{\left[\mu_{+}^{(2)}\left(\frac{27}{2}+9 \nu\right)+\frac{45}{2} \Delta \mu_{-}^{(2)}-24 \sigma_{+}^{(2)}\right] \dot{r}^{2}+\left[\mu_{+}^{(2)}\left(\frac{15}{4}+\frac{3}{2} \nu\right)-\frac{15}{4} \Delta \mu_{-}^{(2)}\right.\right.\right. \\
& \left.\left.+24 \sigma_{+}^{(2)}\right] v^{2}+\frac{G m}{r}\left(-\frac{27}{2} \mu_{+}^{(2)}+\frac{15}{2} \Delta \mu_{-}^{(2)}\right)\right\}+\frac{1}{c^{4}}\left[r \left\{\left[\mu_{+}^{(2)}\left(21-\frac{45}{2} \nu\right)+\Delta \mu_{-}^{(2)}\left(21-\frac{9}{2} \nu\right)\right.\right.\right. \\
& \left.-48 \nu \sigma_{+}^{(2)}\right] a_{v} \dot{r}+\left[\mu_{+}^{(2)}(-60+18 \nu)+\Delta \mu_{-}^{(2)}(-60+18 \nu)+\sigma_{+}^{(2)}(-16+48 \nu)-16 \Delta \sigma_{-}^{(2)}\right] a_{n} \dot{r}^{2} \\
& \left.+\left[\mu_{+}^{(2)}\left(\frac{39}{2}-\frac{27}{4} \nu\right)+\Delta \mu_{-}^{(2)}\left(\frac{39}{2}-\frac{9}{4} \nu\right)+16 \sigma_{+}^{(2)}+16 \Delta \sigma_{-}^{(2)}\right] a_{n} v^{2}\right\}+\left[\mu_{+}^{(2)}\left(36-72 \nu+18 \nu^{2}\right)\right. \\
& \left.+\Delta \mu_{-}^{(2)}(27-18 \nu)+\sigma_{+}^{(2)}(72-96 \nu)+48 \Delta \sigma_{-}^{(2)}\right] \dot{r}^{4}+\left[\mu_{+}^{(2)}\left(-\frac{189}{4}+72 \nu-\frac{45}{2} \nu^{2}\right)+\Delta \mu_{-}^{(2)}\left(-\frac{99}{4}\right.\right. \\
& \left.\left.-\frac{27}{2} \nu\right)+\sigma_{+}^{(2)}(-114+132 \nu)-54 \Delta \sigma_{-}^{(2)}\right] \dot{r}^{2} v^{2}+\left[\mu_{+}^{(2)}\left(\frac{249}{16}-12 \nu-\frac{27}{8} \nu^{2}\right)+\Delta \mu_{-}^{(2)}\left(\frac{39}{16}+\frac{27}{8} \nu\right)\right. \\
& \left.+\sigma_{+}^{(2)}(42-36 \nu)+6 \Delta \sigma_{-}^{(2)}\right] v^{4}+\frac{G m}{r}\left\{\left[\mu_{+}^{(2)}\left(-\frac{249}{2}+\frac{355}{2} \nu+39 \nu^{2}\right)+\Delta \mu_{-}^{(2)}\left(-\frac{303}{2}+\frac{135}{2} \nu\right)\right.\right. \\
& \left.\left.+28 \sigma_{+}^{(2)}-44 \Delta \sigma_{-}^{(2)}\right] \dot{r}^{2}+\left[\mu_{+}^{(2)}\left(\frac{123}{4}-41 \nu+3 \nu^{2}\right)+\frac{213}{4} \Delta \mu_{-}^{(2)}-28 \sigma_{+}^{(2)}+44 \Delta \sigma_{-}^{(2)}\right] v^{2}\right\} \\
& \left.\left.+\frac{G^{2} m^{2}}{r^{2}}\left[\mu_{+}^{(2)}\left(\frac{915}{28}+\frac{3119}{28} \nu\right)-\frac{1395}{28} \Delta \mu_{-}^{(2)}\right]\right]+\mu_{+}^{(3)} \frac{15}{r^{2}}\right\}+\mathcal{O}\left(\frac{\epsilon_{\text {tidal }}}{c^{6}}\right) .
\end{aligned}
$$

Note again that the last term is actually a NNL/2PN contribution. The corresponding relative CoM acceleration is displayed in Appendix C. Similarly, we show here the tidal part of the conserved energy $E=E_{\mathrm{pp}}+E_{\mathrm{tidal}}$ :

$$
\begin{aligned}
\frac{E_{\text {tidal }}}{m \nu}= & -3 \frac{G^{2} m}{r^{6}} \mu_{+}^{(2)}+\frac{1}{c^{2}}\left\{\frac { G ^ { 2 } m } { r ^ { 6 } } \left[\left[\left(\frac{27}{2}+9 \nu\right) \mu_{+}^{(2)}+\frac{45}{2} \Delta \mu_{-}^{(2)}-24 \sigma_{+}^{(2)}\right] \dot{r}^{2}\right.\right. \\
& \left.\left.+\left(\left(\frac{15}{4}+\frac{3}{2} \nu\right) \mu_{+}^{(2)}-\frac{15}{4} \Delta \mu_{-}^{(2)}+24 \sigma_{+}^{(2)}\right) v^{2}\right]+\frac{G^{3} m^{2}}{r^{7}}\left[\frac{27}{2} \mu_{+}^{(2)}-\frac{15}{2} \Delta \mu_{-}^{(2)}\right]\right\} \\
& +\frac{1}{c^{4}}\left\{\frac { G ^ { 2 } m } { r ^ { 6 } } \left[\left(\left(-372-72 \nu+54 \nu^{2}\right) \mu_{+}^{(2)}+(-399+90 \nu) \Delta \mu_{-}^{(2)}+(88+96 \nu) \sigma_{+}^{(2)}+16 \Delta \sigma_{-}^{(2)}\right) \dot{r}^{4}\right.\right. \\
& +\left(\left(\frac{1125}{4}-\frac{27}{2} \nu-\frac{135}{2} \nu^{2}\right) \mu_{+}^{(2)}+\left(\frac{1395}{4}-135 \nu\right) \Delta \mu_{-}^{(2)}+(-198-36 \nu) \sigma_{+}^{(2)}-18 \Delta \sigma_{-}^{(2)}\right) \dot{r}^{2} v^{2} \\
& \left.+\left(\left(\frac{99}{16}-\frac{27}{4} \nu-\frac{81}{8} \nu^{2}\right) \mu_{+}^{(2)}+\left(-\frac{531}{16}+\frac{135}{8} \nu\right) \Delta \mu_{-}^{(2)}+(110-60 \nu) \sigma_{+}^{(2)}+2 \Delta \sigma_{-}^{(2)}\right) v^{4}\right] \\
& +\frac{G^{3} m^{2}}{r^{7}}\left[\left(\left(-\frac{213}{2}+\frac{499}{2} \nu+39 \nu^{2}\right) \mu_{+}^{(2)}+\left(-\frac{267}{2}+\frac{135}{2} \nu\right) \Delta \mu_{-}^{(2)}+(60+48 \nu) \sigma_{+}^{(2)}-12 \Delta \sigma_{-}^{(2)}\right) \dot{r}^{2}\right. \\
& \left.+\left(\left(\frac{51}{4}-113 \nu+3 \nu^{2}\right) \mu_{+}^{(2)}+\frac{141}{4} \Delta \mu_{-}^{(2)}+(-60-48 \nu) \sigma_{+}^{(2)}+12 \Delta \sigma_{-}^{(2)}\right) v^{2}\right]
\end{aligned}
$$




$$
\left.+\frac{G^{4} m^{3}}{r^{8}}\left[\left(-\frac{915}{28}-\frac{3119}{28} \nu\right) \mu_{+}^{(2)}+\frac{1395}{28} \Delta \mu_{-}^{(2)}\right]\right\}-15 \frac{G^{2} m}{r^{8}} \mu_{+}^{(3)}+\mathcal{O}\left(\frac{\epsilon_{\text {tidal }}}{c^{6}}\right) .
$$

Finally, for the CoM angular momentum $J^{i}=J_{\mathrm{pp}}^{i}+J_{\text {tidal }}^{i}$, we find (denoting $\left.L^{i}=\varepsilon_{i j k} x^{j} v^{k}\right)$

$$
\begin{aligned}
\frac{J_{\text {tidal }}^{i}}{m \nu} & =\frac{G^{2} m}{c^{2} r^{6}} L^{i}\left[\mu_{+}^{(2)}\left(\frac{15}{2}+3 \nu\right)-\frac{15}{2} \Delta \mu_{-}^{(2)}+48 \sigma_{+}^{(2)}+\frac{1}{c^{2}}\left\{\left[\mu_{+}^{(2)}\left(\frac{303}{2}-27 \nu-45 \nu^{2}\right)+\Delta \mu_{-}^{(2)}\left(\frac{393}{2}-90 \nu\right)\right.\right.\right. \\
& \left.+\sigma_{+}^{(2)}(-196-120 \nu)-76 \Delta \sigma_{-}^{(2)}\right] \dot{r}^{2}+\left[\mu_{+}^{(2)}\left(\frac{9}{4}-12 \nu-\frac{27}{2} \nu^{2}\right)+\Delta \mu_{-}^{(2)}\left(-\frac{201}{4}+\frac{45}{2} \nu\right)+\sigma_{+}^{(2)}(136\right. \\
& \left.\left.\left.-96 \nu)-8 \Delta \sigma_{-}^{(2)}\right] v^{2}+\frac{G m}{r}\left[\mu_{+}^{(2)}\left(\frac{87}{2}-154 \nu+6 \nu^{2}\right)+\frac{177}{2} \Delta \mu_{-}^{(2)}+\sigma_{+}^{(2)}(-88-48 \nu)+56 \Delta \sigma_{-}^{(2)}\right]\right\}\right] \\
& +\mathcal{O}\left(\frac{\epsilon_{\text {tidal }}}{c^{6}}\right) .
\end{aligned}
$$

The point-particle pieces $E_{\mathrm{pp}}$ and $J_{\mathrm{pp}}^{i}$ are depicted in Eqs. (4.8) and (4.9) of Ref. [45].

\section{TIDAL EFFECTS FOR QUASI-CIRCULAR ORBITS}

We consider quasi-circular orbits, i.e. orbits that are circular in our harmonic coordinate system but for the dissipative radiation-reaction effects. For such orbits, we can neglect $\dot{r}=\mathcal{O}\left(c^{-5}\right)$, which is precisely of the order of radiation reaction effects. Under this assumption, we see from Eq. $(\mathrm{C} 2)$ that the CoM acceleration becomes purely radial, $a^{i}=-\omega^{2} x^{i}$, from which we can read off the orbital angular frequency $\omega$. Relevant quantities will then depend only on the bodies' separation $r$ or, equivalently (via a generalized Kepler third law), on the orbital frequency $\omega$. In the case of circular orbits, it is convenient to introduce the dimensionless PN parameters associated with the separation and orbital frequency as

$$
\gamma=\frac{G m}{r c^{2}}, \quad x=\left(\frac{G m \omega}{c^{3}}\right)^{2 / 3}
$$

as well as to adimensionalize the polarizability coefficients defined in Eqs. (5.3) by considering the "tilded" quantities ${ }^{8}$

$$
\widetilde{\mu}_{ \pm}^{(\ell)}=\left(\frac{c^{2}}{G m}\right)^{2 \ell+1} G \mu_{ \pm}^{(\ell)}, \quad \widetilde{\sigma}_{ \pm}^{(\ell)}=\left(\frac{c^{2}}{G m}\right)^{2 \ell+1} G \sigma_{ \pm}^{(\ell)}
$$

By identifying the expression of $\omega^{2}$ from the circular-orbit EoM as explained above and replacing $\gamma$ iteratively, we recover the well-known formula for point masses at the $2 \mathrm{PN}$ order, with a non-trivial NNL/2PN relative tidal contribution

$$
\begin{aligned}
\left(\omega^{2}\right)_{\mathrm{pp}}=\frac{G m}{r^{3}}\left[1+(-3+\nu) \gamma+\left(6+\frac{61}{4} \nu+\nu^{2}\right) \gamma^{2}\right]+\mathcal{O}\left(\frac{1}{c^{6}}\right) \\
\left(\omega^{2}\right)_{\text {tidal }}=\frac{G m}{r^{3}}\left\{18 \widetilde{\mu}_{+}^{(2)} \gamma^{5}+\left[\left(-\frac{249}{2}+51 \nu\right) \widetilde{\mu}_{+}^{(2)}+\frac{75}{2} \Delta \widetilde{\mu}_{-}^{(2)}+96 \widetilde{\sigma}_{+}^{(2)}\right] \gamma^{6}\right. \\
+\left[\left(\frac{34317}{56}+\frac{2976}{7} \nu+54 \nu^{2}\right) \widetilde{\mu}_{+}^{(2)}+\left(-\frac{12051}{56}+90 \nu\right) \Delta \widetilde{\mu}_{-}^{(2)}\right. \\
\left.\left.+(-616+264 \nu) \widetilde{\sigma}_{+}^{(2)}+200 \Delta \widetilde{\sigma}_{-}^{(2)}+120 \widetilde{\mu}_{+}^{(3)}\right] \gamma^{7}\right\}+\mathcal{O}\left(\frac{\epsilon_{\text {tidal }}}{c^{6}}\right) .
\end{aligned}
$$

Next, we may determine the relation between $\gamma$ and $x$, defined in Eqs. (6.1), by inverting Eqs. (6.3), with result:

$$
\gamma_{\mathrm{pp}}=x\left[1+\left(1-\frac{\nu}{3}\right) x+\left(1-\frac{65}{12} \nu\right) x^{2}\right]+\mathcal{O}\left(\frac{1}{c^{6}}\right)
$$

\footnotetext{
${ }^{8}$ The quantity $\kappa_{2}^{T}$ defined in Ref. [7] is related to our definition $\widetilde{\mu}_{+}^{(2)}$ by $\kappa_{2}^{T}=6 \widetilde{\mu}_{+}^{(2)}$.
} 


$$
\begin{aligned}
\gamma_{\text {tidal }}=x\left\{-6 \widetilde{\mu}_{+}^{(2)} x^{5}+\left[\left(-\frac{37}{2}+3 \nu\right) \widetilde{\mu}_{+}^{(2)}-\frac{25}{2} \Delta \widetilde{\mu}_{-}^{(2)}-32 \widetilde{\sigma}_{+}^{(2)}\right] x^{6}\right. \\
+\left[\left(-\frac{4355}{56}+\frac{1105}{21} \nu+15 \nu^{2}\right) \widetilde{\mu}_{+}^{(2)}+\left(-\frac{3683}{56}+\frac{95}{6} \nu\right) \Delta \widetilde{\mu}_{-}^{(2)}\right. \\
\left.\left.\quad+\left(-\frac{440}{3}+\frac{88}{3} \nu\right) \widetilde{\sigma}_{+}^{(2)}-\frac{200}{3} \Delta \widetilde{\sigma}_{-}^{(2)}-40 \widetilde{\mu}_{+}^{(3)}\right] x^{7}\right\}+\mathcal{O}\left(\frac{\epsilon_{\text {tidal }}}{c^{6}}\right) .
\end{aligned}
$$

The conserved energy for circular orbits can now be computed. To do so, we take Eq. (5.5) to which we add the point-particle part, set $\dot{r}=0$ and replace $v^{2}=r^{2} \omega^{2}$ by its expression in terms of the parameter $\gamma$ using Eqs. (6.3). This yields $E$ first as a function of $\gamma$. We finally insert there the previous relation (6.4) between $\gamma$ and $x$ to get an important result, namely the expression of the circular energy as a function of the frequency-dependent parameter $x$ :

$$
\begin{aligned}
& E_{\mathrm{pp}}=-\frac{1}{2} m \nu x c^{2} {\left[1+\left(-\frac{3}{4}-\frac{\nu}{12}\right) x+\left(-\frac{27}{8}+\frac{19}{8} \nu-\frac{\nu^{2}}{24}\right) x^{2}\right]+\mathcal{O}\left(\frac{1}{c^{6}}\right) } \\
& E_{\text {tidal }}=-\frac{1}{2} m \nu x c^{2}\left\{-18 \widetilde{\mu}_{+}^{(2)} x^{5}+\left[\left(-\frac{121}{2}+33 \nu\right) \widetilde{\mu}_{+}^{(2)}-\frac{55}{2} \Delta \widetilde{\mu}_{-}^{(2)}-176 \widetilde{\sigma}_{+}^{(2)}\right] x^{6}\right. \\
&+\left[\left(-\frac{20865}{56}+\frac{5434}{21} \nu-\frac{91}{4} \nu^{2}\right) \widetilde{\mu}_{+}^{(2)}+\Delta\left(-\frac{11583}{56}+\frac{715}{12} \nu\right) \widetilde{\mu}_{-}^{(2)}\right. \\
&\left.\left.+\left(-\frac{2444}{3}+\frac{1768}{3} \nu\right) \widetilde{\sigma}_{+}^{(2)}-\frac{884}{3} \Delta \widetilde{\sigma}_{-}^{(2)}-130 \widetilde{\mu}_{+}^{(3)}\right] x^{7}\right\}+\mathcal{O}\left(\frac{\epsilon_{\text {tidal }}}{c^{6}}\right) .
\end{aligned}
$$

We can also compute by the same method the constant angular momentum for circular orbits, which reads

$$
\begin{aligned}
J_{\mathrm{pp}}=\frac{G m^{2} \nu}{c x^{1 / 2}} & {\left[1+\left(\frac{3}{2}+\frac{\nu}{6}\right) x+\left(\frac{27}{8}-\frac{19}{8} \nu+\frac{\nu^{2}}{24}\right) x^{2}\right]+\mathcal{O}\left(\frac{1}{c^{6}}\right) } \\
J_{\text {tidal }}=\frac{G m^{2} \nu}{c x^{1 / 2}} & \left\{12 \widetilde{\mu}_{+}^{(2)} x^{5}+\left[\left(\frac{77}{2}-21 \nu\right) \widetilde{\mu}_{+}^{(2)}+\frac{35}{2} \Delta \widetilde{\mu}_{-}^{(2)}+112 \widetilde{\sigma}_{+}^{(2)}\right] x^{6}+\left[\left(\frac{1605}{7}-\frac{3344}{21} \nu+14 \nu^{2}\right) \widetilde{\mu}_{+}^{(2)}\right.\right. \\
& \left.\left.+\Delta\left(\frac{891}{7}-\frac{110}{3} \nu\right) \widetilde{\mu}_{-}^{(2)}+\left(\frac{1504}{3}-\frac{1088}{3} \nu\right) \widetilde{\sigma}_{+}^{(2)}+\frac{544}{3} \Delta \widetilde{\sigma}_{-}^{(2)}+80 \widetilde{\mu}_{+}^{(3)}\right] x^{7}\right\}+\mathcal{O}\left(\frac{\epsilon_{\text {tidal }}}{c^{6}}\right) .
\end{aligned}
$$

We have verified that the energy $E$ and angular momentum $J$ for circular orbits, including all the tidal contributions given in (6.5)-(6.6), are linked by the famous relation

$$
\frac{\partial E}{\partial \omega}=\omega \frac{\partial \mathrm{J}}{\partial \omega}+\mathcal{O}\left(\frac{1}{c^{6}}, \frac{\epsilon_{\text {tidal }}}{c^{6}}\right),
$$

which is just one aspect of the "first law of binary point-particle mechanics" [46].

\section{SUMMARY AND CONCLUSIONS}

We have computed the Lagrangian and associated conserved quantities of compact binaries including tidal interactions up to the NNL order, corresponding to the $2 \mathrm{PN}$ approximation beyond the leading quadrupolar tidal effect occuring at the 5PN order. The results follow from the effective Fokker action (2.1), (2.2) with non-minimal matter couplings, and are parametrized by polarizability coefficients describing the mass quadrupole, mass octupole and current quadrupole tidal interactions. In particular, we have obtained the NNL conserved invariant energy of the compact binary for quasi-circular orbits.

To conclude, let us compare our expressions for the invariant energy as given by (6.5) with existing results in the literature. In the following table, we provide for each order and for each multipolar piece contributing to the conserved energy $E_{\text {tidal }}(x)$ the references which we agree with:

\begin{tabular}{|c||c|c|c|}
\hline$E_{\text {tidal }}$ & Mass quadrupole & Current quadrupole & Mass octupole \\
\hline \hline $5 \mathrm{PN}(\mathrm{L})$ & {$[6,8,18-20]$} & $\times$ & $\times$ \\
\hline $6 \mathrm{PN}(\mathrm{NL})$ & {$[19-21]$} & {$[19,21,22]$} & $\times$ \\
\hline $7 \mathrm{PN}(\mathrm{NNL})$ & {$[19]$} & {$[19]$} & {$[19,23]$} \\
\hline
\end{tabular}


Note in particular that we are in full agreement with all results of Ref. [19]. We have checked, notably, that by re-expanding the tidal effects entering the EOB Hamiltonian [19] in the form of a PN Taylor series, we recover exactly our equation $(6.5) .^{9}$

Now that the problem of the Lagrangian and EoM is solved (Ref. [19] and this work), we shall compute in a second paper [24] the gravitational-wave energy flux for quasi-circular orbits, and then, from it, deduce, through the energy balance equation, the crucial orbital phase and frequency evolution (or "chirp") of compact binaries in circular orbits including tidal effects up to the NNL/2PN order beyond the Einstein quadrupole formula.

\section{ACKNOWLEDGMENTS}

We thank Gilles Esposito-Farèse for useful discussions. We are also greatful to Justin Vines for interesting informative discussions during the preparation of this work.

\section{Appendix A: Newtonian treatment of the tidal effects}

In this Appendix, we derive the Newtonian EoM and the Lagrangian of a system of $N$ extended compact bodies without spins, including multipolar tidal interaction effects. The mass and the CoM position of each of the objects are defined by

$$
m_{A}=\int_{\mathcal{V}_{A}} \mathrm{~d}^{3} \mathbf{x} \rho(\mathbf{x}, t), \quad y_{A}^{i}(t)=\frac{1}{m_{A}} \int_{\mathcal{V}_{A}} \mathrm{~d}^{3} \mathbf{x} \rho(\mathbf{x}, t) x^{i}
$$

where the integrals extend over the volume $\mathcal{V}_{A}$ of body $A$, and where $\rho(\mathbf{x}, t)$ denotes the Eulerian density of the $N$-body system satisfying the usual continuity equation $\partial_{t} \rho+\partial_{i}\left(\rho v^{i}\right)=0$ (hence the mass $m_{A}$ is constant). The equation of motion verified by the CoM line of body $A$ is then given by

$$
m_{A} \frac{\mathrm{d}^{2} y_{A}^{i}}{\mathrm{~d} t^{2}}=\sum_{B \neq A} \int_{\mathcal{V}_{A}} \mathrm{~d}^{3} \mathbf{x} \rho \partial_{i} U_{B}
$$

where we have discarded the self-field of body $A$ which is zero by Newton's action-reaction theorem (so the sum runs over all the bodies $B \neq A$ ), and where the Newtonian potential generated by body $B$ reads

$$
U_{B}(\mathbf{x}, t)=G \int_{\mathcal{V}_{B}} \frac{\mathrm{d}^{3} \mathbf{x}^{\prime}}{\left|\mathbf{x}-\mathbf{x}^{\prime}\right|} \rho\left(\mathbf{x}^{\prime}, t\right) .
$$

For any point outside the body $B$, thus in particular located inside the body $A$, distinct from $B$, we have $\Delta U_{B}=0$. Next, we define the Newtonian STF multipole moment of body $A$ to be

$$
I_{A}^{L}(t)=\int_{\mathcal{V}_{A}} \mathrm{~d}^{3} \boldsymbol{z}_{A} \rho_{A}\left(\boldsymbol{z}_{A}, t\right) \hat{z}_{A}^{L},
$$

where we adopted as integration variable the distance $\boldsymbol{z}_{A}=\mathbf{x}-\boldsymbol{y}_{A}(t)$ linking the line of the CoM $\boldsymbol{y}_{A}(t)$ to the generic point $\mathbf{x} \in \mathcal{V}_{A}$, where $\hat{z}_{A}^{L}=\operatorname{STF}\left(z_{A}^{L}\right)$ denotes the STF product of $\ell$ spatial vectors $z_{A}^{L}=z_{A}^{i_{1}} \cdots z_{A}^{i_{\ell}}$ (with $L=i_{1} \cdots i_{\ell}$ a multi-spatial index), and where we have posed $\rho_{A}\left(\boldsymbol{z}_{A}, t\right)=\rho\left(\boldsymbol{y}_{a}+\boldsymbol{z}_{a}, t\right)$. With this notation the mass monopole moment is just the constant mass, while the CoM position $y_{A}^{i}$ is defined by the nullity of the mass dipole moment:

$$
I_{A}=m_{A}, \quad I_{A}^{i}=0 .
$$

On the other hand, the Newtonian tidal moments, starting with the quadrupole moment $(\ell \geqslant 2)$, are defined quite naturally as the multi-gradients of the total external potential due to the other bodies felt by the body $A$ at the location of its $\mathrm{CoM} \boldsymbol{y}_{A}$ :

$$
G_{A}^{L}(t)=\sum_{B \neq A}\left(\partial_{L} U_{B}\right)\left(\boldsymbol{y}_{A}\right) \quad(\text { for } \ell \geqslant 2),
$$

\footnotetext{
${ }^{9}$ However, we do not recover the 1PN coefficient for the current quadrupole piece in Ref. [23], where the discrepancy is by a factor 2 .
} 
with $\partial_{L}=\partial_{i_{1}} \cdots \partial_{i_{\ell}}$. Since $\Delta U_{B}=0$ inside body $A$, the tidal moments are automatically STF in all their indices $L$, namely $\partial_{L} U_{B}=\hat{\partial}_{L} U_{B}$. For the dipolar tidal moment (with $\ell=1$ ) it is convenient to pose

$$
G_{A}^{i}=\sum_{B \neq A}\left(\partial_{i} U_{B}\right)\left(\boldsymbol{y}_{A}\right)-\frac{\mathrm{d}^{2} y_{A}^{i}}{\mathrm{~d} t^{2}}
$$

so that $G_{A}^{i}=0$ for a system of point particles described only by their masses, their higher multipole moments being neglected. The EoM may then be rewritten in elegant form as (see e.g. [32])

$$
m_{A} G_{A}^{i}+\sum_{\ell=2}^{+\infty} \frac{1}{\ell !} I_{A}^{L} G_{A}^{i L}=0
$$

Using the fact that for any $\mathbf{x}$ outside the body $B$ we have the multipole decomposition

$$
U_{B}=G \sum_{k=0}^{+\infty} \frac{(-)^{k}}{k !} I_{B}^{K} \partial_{K}\left(\frac{1}{r_{B}}\right),
$$

with $r_{B}=\left|\mathbf{x}-\boldsymbol{y}_{B}\right|$, we see that the tidal moments themselves (A6) can be expanded in terms of the multipole moments of the other bodies as (for $\ell \geqslant 2$ )

$$
G_{A}^{L}=G \sum_{B \neq A} \sum_{k=0}^{+\infty} \frac{(-)^{k}}{k !} I_{B}^{K} \partial_{L K}^{A}\left(\frac{1}{r_{A B}}\right),
$$

where $r_{A B}=\left|\boldsymbol{y}_{A}-\boldsymbol{y}_{B}\right|$ is the distance between the CoMs of the bodies $A$ and $B$, the gradient is taken with respect to the point $A$, i.e. $\partial_{i}^{A}=\partial / \partial y_{A}^{i}$, and we denote $\partial_{L K}^{A}=\partial_{L}^{A} \partial_{K}^{A}$ with $\partial_{L}^{A}=\partial_{i_{1}}^{A} \cdots \partial_{i_{\ell}}^{A}$. Finally, the EoM admit the double multipole expansion series

$$
m_{A} \frac{\mathrm{d}^{2} y_{A}^{i}}{\mathrm{~d} t^{2}}=G \sum_{B \neq A} \sum_{\ell=0}^{+\infty} \sum_{k=0}^{+\infty} \frac{(-)^{k}}{\ell ! k !} I_{A}^{L} I_{B}^{K} \partial_{i L K}^{A}\left(\frac{1}{r_{A B}}\right),
$$

or in more details (see e.g. Eq. (1.201) of [47]),

$$
\begin{aligned}
m_{A} \frac{\mathrm{d}^{2} y_{A}^{i}}{\mathrm{~d} t^{2}}=G \sum_{B \neq A}\left\{m_{A} m_{B} \partial_{i}^{A}\left(\frac{1}{r_{A B}}\right)\right. & +\sum_{\ell=2}^{+\infty} \frac{(-)^{\ell}}{\ell !}\left[m_{A} I_{B}^{L}+(-)^{\ell} m_{B} I_{A}^{L}\right] \partial_{i L}^{A}\left(\frac{1}{r_{A B}}\right) \\
& \left.+\sum_{\ell=2}^{+\infty} \sum_{k=2}^{+\infty} \frac{(-)^{k}}{\ell ! k !} I_{A}^{L} I_{B}^{K} \partial_{i L K}^{A}\left(\frac{1}{r_{A B}}\right)\right\} .
\end{aligned}
$$

Those equations have been generalized to the 1PN order [20, 48-50] using the DSX formalism [32, 51].

We now consider the case where the multipole moments are exclusively induced by the tidal field of the other bodies. To describe this situation, we assume that each extended body is at hydrodynamical equilibrium at every time, so that the mass distribution at any instant is aligned on the equipotentials of the external gravitational field. We are thus in the so-called adiabatic regime where the relaxation time scale of the body internal dynamics is significantly smaller than the orbital time scale. In particular, we neglect the dissipative effects due to the tides, considering only the conservative dynamics of the system, and look for a Lagrangian. In this case, we introduce a linear-response coefficient $\mu^{(\ell)}$ depending on the internal structure of the body and characterizing its deformability or "polarizability" under the influence of the external field, such that its multipole moments obey

$$
I_{A}^{L}=\mu_{A}^{(\ell)} G_{A}^{L} .
$$

Following usual definitions (see e.g. $[10,13,14]$ ), this coefficient is related to the radius $R$ of the body and the (mass-type) multipolar Love numbers $k^{(\ell)}$ by

$$
G \mu_{A}^{(\ell)}=\frac{2}{(2 \ell-1) ! !} k_{A}^{(\ell)} R_{A}^{2 \ell+1} .
$$


The Newtonian EoM (A12) become now

$$
\begin{aligned}
m_{A} \frac{\mathrm{d}^{2} y_{A}^{i}}{\mathrm{~d} t^{2}}=G \sum_{B \neq A}\left\{m_{A} m_{B} \partial_{i}^{A}\left(\frac{1}{r_{A B}}\right)\right. & +\sum_{\ell=2}^{+\infty} \frac{(-)^{\ell}}{\ell !}\left[m_{A} \mu_{B}^{(\ell)} G_{B}^{L}+(-)^{\ell} m_{B} \mu_{A}^{(\ell)} G_{A}^{L}\right] \partial_{i L}^{A}\left(\frac{1}{r_{A B}}\right) \\
& \left.+\sum_{\ell=2}^{+\infty} \sum_{k=2}^{+\infty} \frac{(-)^{k}}{\ell ! k !} \mu_{A}^{(\ell)} \mu_{B}^{(k)} G_{A}^{L} G_{B}^{K} \partial_{i L K}^{A}\left(\frac{1}{r_{A B}}\right)\right\},
\end{aligned}
$$

in which the tidal moments obey the implicit relation

$$
G_{A}^{L}=G \sum_{B \neq A}\left[m_{B} \partial_{L}^{A}\left(\frac{1}{r_{A B}}\right)+\sum_{k=2}^{+\infty} \frac{(-)^{k}}{k !} \mu_{B}^{(k)} G_{B}^{K} \partial_{L K}^{A}\left(\frac{1}{r_{A B}}\right)\right] .
$$

The latter equations describe the conservative dynamics of the system of $N$ extended bodies. The dependence on the internal structure is entirely carried out by the coefficients $\mu^{(\ell)}$, which are supposed to be constant. The dynamics is conservative in the sense that it can be derived from the following exact Lagrangian, valid up to any order in the multipole expansion and the tidal moments:

$$
\begin{aligned}
L & =\sum_{A}\left\{\frac{1}{2} m_{A} v_{A}^{2}+\frac{1}{2} m_{A} \sum_{B \neq A} U_{B}\left(\boldsymbol{y}_{A}\right)\right\} \\
& =\sum_{A}\left\{\frac{1}{2} m_{A} v_{A}^{2}+\frac{1}{2} \sum_{\ell=2}^{+\infty} \frac{1}{\ell !} \mu_{A}^{(\ell)} G_{A}^{L} G_{A}^{L}+G \sum_{B>A}\left[\frac{m_{A} m_{B}}{r_{A B}}-\sum_{\ell=2}^{+\infty} \sum_{k=2}^{+\infty} \frac{(-)^{k}}{\ell ! k !} \mu_{A}^{(\ell)} \mu_{B}^{(k)} G_{A}^{L} G_{B}^{K} \partial_{L K}^{A}\left(\frac{1}{r_{A B}}\right)\right]\right\} .
\end{aligned}
$$

The Newtonian action is formally the Newtonian limit, at the quadratic level, of the non-minimal matter action (2.2) in general relativity. However, the action (2.2) is effective (or "skeletonized"), with each compact object described by an effective point particle endowed with internal structure. The mass-type moments $G^{\hat{L}}$ (even parity sector) entering Eq. (2.2) tend towards the Newtonian tidal moments $G^{L}$, so that they can be regarded as their legitimate relativistic versions, and the corresponding response coefficients $\mu^{(\ell)}$ identify with the Newtonian tidal deformabilities. Moreover, the relativistic action also depends on current-type moments $H^{\hat{L}}$ (odd parity sector) with associated response coefficients $\sigma^{(\ell)}$, first arising at the $1 \mathrm{PN}$ relativistic order.

Both sets of relativistic tidal moments are given by appropriate covariant derivatives of the Riemann tensor, which is nothing but the relativistic tidal field felt by the body. Those moments are evaluated at the location of the particle and a UV-type regularization is required to remove the self field of that particle. Thus, in the effective action, the self-field regularization automatically selects the external tidal field experienced by the body due to the other bodies composing the system.

\section{Appendix B: Proof that the trace terms to NNL order can be removed by a redefinition of the metric}

In this section, we show that the tidal moments entering the action may be defined in terms of the Riemann tensor instead of the Weyl tensor, since the traces of the Riemann tensor do not play any role in the dynamics. Here, we shall denote $G_{\mu \nu}^{(R)}, G_{\lambda \mu \nu}^{(R)}$ and $H_{\mu \nu}^{(R)}$ the tidal mass-quadrupole, mass-octupole and current-quadrupole moments introduced in Eqs. (3.6), while $G_{\mu \nu}^{(C)}, G_{\lambda \mu \nu}^{(C)}$ and $H_{\mu \nu}^{(C)}$ will represent the same but built with the Weyl tensor instead of the Riemann tensor. We thus pose (setting $G=c=1$, and omitting particles' labels and mention of the regularization)

$$
\begin{array}{rrr}
G_{\mu \nu}^{(R)}=-R_{\mu \rho \nu \sigma} u^{\rho} u^{\sigma}, & G_{\mu \nu}^{(C)}=-C_{\mu \rho \nu \sigma} u^{\rho} u^{\sigma}, \\
H_{\mu \nu}^{(R)}=2 R_{(\mu \underline{\rho} \nu) \sigma}^{*} u^{\rho} u^{\sigma}, & H_{\mu \nu}^{(C)}=2 C_{(\mu \underline{\rho} \nu) \sigma}^{*} u^{\rho} u^{\sigma}, \\
G_{\lambda \mu \nu}^{(R)}=-\nabla_{(\lambda}^{\perp} R_{\mu \underline{\rho} \nu) \sigma} u^{\rho} u^{\sigma}, & G_{\lambda \mu \nu}^{(C)}=-\nabla_{(\lambda}^{\perp} C_{\mu \underline{\rho} \nu) \sigma} u^{\rho} u^{\sigma},
\end{array}
$$

where $C_{\mu \nu \rho \sigma}$ stands for the Weyl tensor

$$
C_{\mu \nu \rho \sigma}=R_{\mu \nu \rho \sigma}-\left(g_{\mu[\rho} R_{\sigma] \nu}-g_{\nu[\rho} R_{\sigma] \mu}\right)+\frac{1}{3} g_{\mu[\rho} g_{\sigma] \nu} R,
$$

and where we have used expressions for the original Weyl tidal moments in which the STF operators have been removed or replaced by mere symmetrizations, thanks to the properties of the Weyl tensor and the covariant derivative. To 
start with, we notice that, as one can check, the Riemann and Weyl definitions of the current-type quadrupole coincide, i.e., $H_{\mu \nu}^{(C)}=H_{\mu \nu}^{(R)}$. As a result, the following discussion will, in fact, only concern the mass-type moments. From Eqs. (B1), we then get the following relations:

$$
\begin{aligned}
\left(G_{\mu \nu} G^{\mu \nu}\right)^{(C)} & =\left(G_{\mu \nu} G^{\mu \nu}\right)^{(R)}-G_{(R)}^{\mu \nu} R_{\mu \nu}+(\text { double zero terms }) \\
\left(G_{\mu \nu \rho} G^{\mu \nu \rho}\right)^{(C)} & =\left(G_{\mu \nu \rho} G^{\mu \nu \rho}\right)^{(R)}-G_{(R)}^{\lambda \mu \nu} \nabla_{\lambda} R_{\mu \nu}-\frac{2}{3} u^{\mu} u^{\nu} \nabla^{\kappa} R_{\kappa \mu \lambda \nu}\left[\nabla^{\lambda} R_{\rho \sigma} u^{\rho} u^{\sigma}+\frac{1}{3} \nabla^{\lambda} R\right]+(\text { double zero terms })
\end{aligned}
$$

where the "double zero terms" are terms that are quadratic in the Ricci tensor or scalar. Let us now prove that the actions $S^{(R)}$ and $S^{(C)}$ corresponding to Eq. (2.11) using respectively the Riemann and Weyl definitions, lead to the same EoM.

The double zero terms are treated as follows. Varying their contributions to the action, which have necessarily the general form $\propto \int \mathrm{d}^{4} x \sqrt{-g} A^{\mu \nu \rho \sigma \ldots} \nabla_{\ldots} R_{\mu \nu} \nabla_{\ldots} R_{\rho \sigma}$, leads, after possible integrations by part, to a sum of terms $\propto \int \mathrm{d}^{4} x \sqrt{-g}\left[\nabla_{\ldots}\left(A^{\mu \nu \rho \sigma \ldots} \nabla_{\ldots} R_{\rho \sigma}\right)+\nabla_{\ldots}\left(A^{\rho \sigma \mu \nu \ldots} \nabla_{\ldots} R_{\rho \sigma}\right)\right] \delta R_{\mu \nu}$, plus surface integrals at infinity which vanish, since their integrands contain factors $\nabla \ldots R_{\rho \sigma}$ that are identically zero in vacuum. The remaining terms are then proportional to (the covariant derivatives of) the Ricci tensor multiplied by $A^{\mu \nu \rho \sigma \ldots}$. On the other hand, $A^{\mu \nu \rho \sigma \ldots}$ is itself a sum of the form $\sum_{A} \delta^{(4)}\left(x-y_{A}\right) F_{A}^{\mu \nu \rho \sigma \ldots}$ and the presence of the Dirac distributions forces the evaluation of the Ricci tensor to take place at one particle's location, e.g., at $\boldsymbol{x}=\boldsymbol{y}_{A}$, in the sense of dimensional regularization. Moreover, by virtue of Einstein's equations (reinstalling the particles' label), $\left[R_{\mu \nu}\right]_{A}=8 \pi\left[\left(T_{\mathrm{pp}}\right)_{\mu \nu}-\left(T_{\mathrm{pp}}\right)_{\lambda}^{\lambda} g_{\mu \nu} / 2\right]_{A}+\mathcal{O}\left(\epsilon_{\mathrm{tidal}}\right)$, where $\left[\left(T_{\mathrm{pp}}\right)^{\mu \nu}\right]_{A}$ denotes the point-particle stress-energy tensor of our particle system at point $A$

$$
\left[T_{\mathrm{pp}}^{\mu \nu}\right]_{A}=\sum_{B} m_{B} \int \mathrm{d} \tau_{B} u_{B}^{\mu} u_{B}^{\nu} \frac{\delta^{(4)}\left[y_{A}\left(\tau_{A}\right)-y_{B}\left(\tau_{B}\right)\right]}{\sqrt{-g}}
$$

If $A \neq B$, then $\delta^{(4)}\left[y_{A}\left(\tau_{A}\right)-y_{B}\left(\tau_{B}\right)\right]=0$ because the compact objects never collide in the PN regime. If $A=$ $B$, the Dirac distribution reduces to $\delta^{(4)}(0)$, which is precisely zero in dimensional regularization, as the limit of $\int \mathrm{d}^{d+1} k \mathrm{e}^{2 \pi \mathrm{i} 0}=0$ when $d \rightarrow 3$. Hence $\left[T_{\mathrm{pp}}^{\mu \nu}\right]_{A}$ vanishes as well, and so does the contribution of the double-zero terms to the Euler-Lagrange equations for the point-like bodies.

However, terms that are linear in both the Riemann and the Ricci tensors (or the Ricci scalar) in Eqs. (B3) cannot be dealt with in the same way as the double zeros. Instead, they may be treated by making an appropriate infinitesimal change of variable on the original metric, say $g_{\mu \nu}^{\text {original }}=g_{\mu \nu}+h_{\mu \nu}$, in the action $S^{(C)}\left[g_{\mu \nu}^{\text {original }}, \boldsymbol{y}_{A}\right]$. This naturally defines the new action $\tilde{S}\left[g_{\mu \nu}, \boldsymbol{y}_{A}\right]=S^{(C)}\left[g_{\mu \nu}^{\text {original }}\left[g_{\rho \sigma}, \boldsymbol{y}_{B}\right], \boldsymbol{y}_{A}\right]$, dynamically equivalent to $S^{(C)}$ when regarded as a functional of the metric $g_{\mu \nu}$. At first order in $h_{\mu \nu}$, it reads

$$
\tilde{S}\left[g_{\mu \nu}, \boldsymbol{y}_{A}\right]=S^{(C)}\left[g_{\mu \nu}, \boldsymbol{y}_{A}\right]-\frac{1}{16 \pi} \int \mathrm{d}^{4} x \sqrt{-g}\left(R^{\mu \nu}-\frac{1}{2} R g^{\mu \nu}-8 \pi T^{\mu \nu}\right) h_{\mu \nu}+\mathcal{O}\left(h^{2}\right) .
$$

Now, we want $\tilde{S}\left[g_{\mu \nu}, \boldsymbol{y}_{A}\right]$ to coincide with $S^{(R)}\left[g_{\mu \nu}, \boldsymbol{y}_{A}\right]$. By choosing conveniently $h_{\mu \nu}$, the term $\left(R^{\mu \nu}-\frac{1}{2} R g^{\mu \nu}\right) h_{\mu \nu}$ will cancel the terms linear in the Ricci tensor or scalar entering Eqs. (B3). As for the term $\int \mathrm{d}^{4} x \sqrt{-g} T^{\mu \nu} h_{\mu \nu}$, it vanishes by itself and can thus be ignored. Indeed, integrating the Dirac deltas contained in the expression chosen for $h_{\mu \nu}$ (see below) yields a sum on $A=1,2$ of $\propto\left[T^{\mu \nu}\right]_{A}=\left[T_{\mathrm{pp}}^{\mu \nu}\right]_{A}+\mathcal{O}\left(\epsilon_{\text {tidal }}\right)$, which boils down to $\mathcal{O}\left(\epsilon_{\mathrm{tidal}}\right)$ since $\left[T_{\mathrm{pp}}^{\mu \nu}\right]_{A}=0$, as explained above around Eq. (B4).

Let us examine more precisely how to construct a $h_{\mu \nu}$ suitable to absorb the Ricci-type terms in Eq. (B5) that come from the difference $\Delta G_{\mu \nu}=G_{\mu \nu}^{(C)}-G_{\mu \nu}^{(R)}$. The contribution induced by this difference through the modification of the mass quadrupole invariant $\Delta\left(G_{\mu \nu} G^{\mu \nu}\right)=2 G_{\mu \nu}^{(R)} \Delta G^{\mu \nu}+$ (double zero terms) has the form

$$
\int \mathrm{d}^{4} x Z_{\mu \nu} R^{\mu \nu}=\int \mathrm{d}^{4} x\left(Z_{\mu \nu}-\frac{1}{2} Z_{\lambda}^{\lambda} g_{\mu \nu}\right)\left(R^{\mu \nu}-\frac{1}{2} R g^{\mu \nu}\right) .
$$

It is to be canceled by the piece of the integral in Eq. (B5) that is sourced by $\propto\left(R^{\mu \nu}-\frac{1}{2} R g^{\mu \nu}\right)$. An obvious choice guaranteeing such cancellation is $h_{\mu \nu}^{\left(G_{\rho \sigma}\right)}=16 \pi\left(Z_{\mu \nu}-\frac{1}{2} Z_{\lambda}^{\lambda} g_{\mu \nu}\right) / \sqrt{-g}$. Possible extra terms linear (at least) in the Ricci tensor or scalar merely add irrelevant double zeros to the action. Those can be tuned to have

$$
h_{\mu \nu}^{\left(G_{\rho \sigma}\right)}=-4 \pi \sum_{A} \mu_{A}^{(2)} \int \mathrm{d} \tau_{A}\left[G_{\mu \nu}^{(R)}\right]_{A} \frac{\delta^{(4)}\left[x^{\mu}-y_{A}^{\mu}\left(\tau_{A}\right)\right]}{\sqrt{-g}} .
$$


Regarding the mass octupole, we use the same method as for the mass quadrupole to construct some suitable $h_{\mu \nu}^{\left(G_{\rho \sigma \tau}\right)}$, the only new feature being that $\Delta\left(G_{\lambda \mu \nu} G^{\lambda \mu \nu}\right)$ is now a space-time integral with a source of the form $Z_{\mu \nu}^{\lambda} \nabla_{\lambda} R^{\mu \nu}$. However, the structure (B6) is straightforwardly recovered by integrating by part. We finally find that, in the massoctupolar sector, the equality $\tilde{S}^{\left(G_{\rho \sigma \tau}\right)}=\left(S^{(R)}\right)^{\left(G_{\rho \sigma \tau}\right)}$ is achieved by setting:

$$
h_{\mu \nu}^{\left(G_{\rho \sigma \tau}\right)}=4 \pi \sum_{A} \frac{\mu_{A}^{(3)}}{3} \int \mathrm{d} \tau_{A} \nabla^{\lambda}\left[\left(G_{\lambda \mu \nu}^{(R)}+\frac{2}{3} \nabla^{\kappa} R_{\kappa \rho \lambda \sigma} u^{\rho} u^{\sigma}\left(u_{\mu} u_{\nu}+\frac{2}{3} g_{\mu \nu}\right)\right) \frac{\delta^{(4)}\left[x-y_{A}\left(\tau_{A}\right)\right]}{\sqrt{-g}}\right] .
$$

\section{Appendix C: The tidal acceleration to NNL order}

By varying the total generalized Fokker Lagrangian (4.3)-(4.4) and replacing iteratively the accelerations by the values provided by the EoM consistently truncated at lower orders, we obtain the total acceleration of body 1 as $a_{1}^{i}=\left(a_{1}^{i}\right)_{\mathrm{pp}}+\left(a_{1}^{i}\right)_{\mathrm{tidal}}$, where the point-particle part can be found in e.g. [41], and where

$$
\begin{aligned}
& m_{1}\left(a_{1}^{i}\right)_{\text {tidal }}=\frac{G^{2}}{r_{12}^{7}}\left\{n_{12}^{i}\left(-9 m_{2}^{2} \mu_{1}^{(2)}-9 m_{1}^{2} \mu_{2}^{(2)}\right)+\frac{1}{c^{2}}\left\{n _ { 1 2 } ^ { i } \left[m _ { 2 } ^ { 2 } \mu _ { 1 } ^ { ( 2 ) } \left(-36\left(n_{12} v_{1}\right)^{2}+72\left(n_{12} v_{1}\right)\left(n_{12} v_{2}\right)\right.\right.\right.\right. \\
& \left.-18 v_{12}^{2}+9 v_{1}^{2}\right)+m_{1}^{2} \mu_{2}^{(2)}\left(144\left(n_{12} v_{1}\right)^{2}-288\left(n_{12} v_{1}\right)\left(n_{12} v_{2}\right)+180\left(n_{12} v_{2}\right)^{2}-\frac{81}{2} v_{12}^{2}+9 v_{1}^{2}\right) \\
& +m_{2}^{2} \sigma_{1}^{(2)}\left(-96\left(n_{12} v_{12}\right)^{2}-48 v_{12}^{2}\right)+m_{1}^{2} \sigma_{2}^{(2)}\left(-96\left(n_{12} v_{12}\right)^{2}-48 v_{12}^{2}\right)+\frac{G m_{1}}{r_{12}}\left(\frac{159}{2} m_{2}^{2} \mu_{1}^{(2)}\right. \\
& \left.\left.+132 m_{1}^{2} \mu_{2}^{(2)}\right)+\frac{G m_{2}}{r_{12}}\left(99 m_{2}^{2} \mu_{1}^{(2)}+84 m_{1}^{2} \mu_{2}^{(2)}\right)\right]+v_{1}^{i}\left[m_{2}^{2} \mu_{1}^{(2)}\left(54\left(n_{12} v_{1}\right)-45\left(n_{12} v_{2}\right)\right)\right. \\
& \left.+9 m_{1}^{2} \mu_{2}^{(2)}\left(n_{12} v_{1}\right)+144 m_{2}^{2} \sigma_{1}^{(2)}\left(n_{12} v_{12}\right)+144 m_{1}^{2} \sigma_{2}^{(2)}\left(n_{12} v_{12}\right)\right]+v_{2}^{i}\left[m _ { 2 } ^ { 2 } \mu _ { 1 } ^ { ( 2 ) } \left(-54\left(n_{12} v_{1}\right)\right.\right. \\
& \left.\left.\left.+45\left(n_{12} v_{2}\right)\right)-9 m_{1}^{2} \mu_{2}^{(2)}\left(n_{12} v_{1}\right)-144 m_{2}^{2} \sigma_{1}^{(2)}\left(n_{12} v_{12}\right)-144 m_{1}^{2} \sigma_{2}^{(2)}\left(n_{12} v_{12}\right)\right]\right\} \\
& +\frac{1}{c^{4}}\left[n _ { 1 2 } ^ { i } \left\{m _ { 2 } ^ { 2 } \mu _ { 1 } ^ { ( 2 ) } \left(135\left(n_{12} v_{1}\right)^{4}-540\left(n_{12} v_{1}\right)^{3}\left(n_{12} v_{2}\right)+990\left(n_{12} v_{1}\right)^{2}\left(n_{12} v_{2}\right)^{2}\right.\right.\right. \\
& -900\left(n_{12} v_{1}\right)\left(n_{12} v_{2}\right)^{3}+225\left(n_{12} v_{2}\right)^{4}+72\left(n_{12} v_{1}\right)\left(n_{12} v_{2}\right)\left(v_{1} v_{2}\right)-18\left(v_{1} v_{2}\right)^{2}-126\left(n_{12} v_{1}\right)^{2} v_{12}{ }^{2} \\
& +324\left(n_{12} v_{1}\right)\left(n_{12} v_{2}\right) v_{12}{ }^{2}-90\left(n_{12} v_{2}\right)^{2} v_{12}{ }^{2}-36\left(v_{1} v_{2}\right) v_{12}{ }^{2}-27 v_{12}{ }^{4}-72\left(n_{12} v_{1}\right)\left(n_{12} v_{2}\right) v_{1}{ }^{2} \\
& \left.+36\left(v_{1} v_{2}\right) v_{1}^{2}+36 v_{12}^{2} v_{1}^{2}-18 v_{1}^{4}\right)+m_{1}^{2} \mu_{2}^{(2)}\left(-3855\left(n_{12} v_{1}\right)^{4}+15420\left(n_{12} v_{1}\right)^{3}\left(n_{12} v_{2}\right)\right. \\
& -23850\left(n_{12} v_{1}\right)^{2}\left(n_{12} v_{2}\right)^{2}+16860\left(n_{12} v_{1}\right)\left(n_{12} v_{2}\right)^{3}-4665\left(n_{12} v_{2}\right)^{4}-288\left(n_{12} v_{1}\right)\left(n_{12} v_{2}\right)\left(v_{1} v_{2}\right) \\
& +360\left(n_{12} v_{2}\right)^{2}\left(v_{1} v_{2}\right)-\frac{81}{2}\left(v_{1} v_{2}\right)^{2}+2598\left(n_{12} v_{1}\right)^{2} v_{12}^{2}-5484\left(n_{12} v_{1}\right)\left(n_{12} v_{2}\right) v_{12}^{2} \\
& +3084\left(n_{12} v_{2}\right)^{2} v_{12}{ }^{2}-81\left(v_{1} v_{2}\right) v_{12}{ }^{2}-\frac{1923}{8} v_{12}{ }^{4}+288\left(n_{12} v_{1}\right)\left(n_{12} v_{2}\right) v_{1}{ }^{2}-360\left(n_{12} v_{2}\right)^{2} v_{1}^{2} \\
& \left.+81\left(v_{1} v_{2}\right) v_{1}^{2}+81 v_{12}^{2} v_{1}^{2}-\frac{81}{2} v_{1}^{4}\right)+m_{2}^{2} \sigma_{1}^{(2)}\left(840\left(n_{12} v_{12}\right)^{4}-960\left(n_{12} v_{12}\right)^{3}\left(n_{12} v_{1}\right)\right. \\
& +480\left(n_{12} v_{12}\right)^{2}\left(n_{12} v_{1}\right)^{2}-192\left(n_{12} v_{12}\right)^{2}\left(v_{1} v_{2}\right)+192\left(n_{12} v_{12}\right)\left(n_{12} v_{1}\right)\left(v_{1} v_{2}\right)-48\left(v_{1} v_{2}\right)^{2} \\
& -336\left(n_{12} v_{12}\right)^{2} v_{12}{ }^{2}-192\left(n_{12} v_{12}\right)\left(n_{12} v_{1}\right) v_{12}{ }^{2}+192\left(n_{12} v_{1}\right)^{2} v_{12}{ }^{2}-96\left(v_{1} v_{2}\right) v_{12}{ }^{2}-72 v_{12}{ }^{4} \\
& \left.+192\left(n_{12} v_{12}\right)^{2} v_{1}^{2}-192\left(n_{12} v_{12}\right)\left(n_{12} v_{1}\right) v_{1}^{2}+96\left(v_{1} v_{2}\right) v_{1}^{2}+96 v_{12}^{2} v_{1}^{2}-48 v_{1}^{4}\right) \\
& +m_{1}^{2} \sigma_{2}^{(2)}\left(1000\left(n_{12} v_{12}\right)^{4}-960\left(n_{12} v_{12}\right)^{3}\left(n_{12} v_{1}\right)+480\left(n_{12} v_{12}\right)^{2}\left(n_{12} v_{1}\right)^{2}-192\left(n_{12} v_{12}\right)^{2}\left(v_{1} v_{2}\right)\right. \\
& +192\left(n_{12} v_{12}\right)\left(n_{12} v_{1}\right)\left(v_{1} v_{2}\right)-48\left(v_{1} v_{2}\right)^{2}+64\left(n_{12} v_{12}\right)^{2} v_{12}{ }^{2}-192\left(n_{12} v_{12}\right)\left(n_{12} v_{1}\right) v_{12}{ }^{2} \\
& +192\left(n_{12} v_{1}\right)^{2} v_{12}{ }^{2}-96\left(v_{1} v_{2}\right) v_{12}{ }^{2}-128 v_{12}{ }^{4}+192\left(n_{12} v_{12}\right)^{2} v_{1}^{2}-192\left(n_{12} v_{12}\right)\left(n_{12} v_{1}\right) v_{1}^{2} \\
& \left.+96\left(v_{1} v_{2}\right) v_{1}^{2}+96 v_{12}^{2} v_{1}^{2}-48 v_{1}^{4}\right)+\frac{G m_{1}}{r_{12}}\left[m _ { 2 } ^ { 2 } \mu _ { 1 } ^ { ( 2 ) } \left(\frac{7215}{8}\left(n_{12} v_{1}\right)^{2}-\frac{7431}{4}\left(n_{12} v_{1}\right)\left(n_{12} v_{2}\right)\right.\right. \\
& \left.+\frac{4461}{8}\left(n_{12} v_{2}\right)^{2}-\frac{285}{8} v_{12}^{2}-\frac{159}{2} v_{1}^{2}\right)+m_{1}^{2} \mu_{2}^{(2)}\left(-\frac{15717}{8}\left(n_{12} v_{1}\right)^{2}+\frac{16581}{4}\left(n_{12} v_{1}\right)\left(n_{12} v_{2}\right)\right.
\end{aligned}
$$




$$
\begin{aligned}
& \left.-\frac{22521}{8}\left(n_{12} v_{2}\right)^{2}+\frac{4597}{8} v_{12}^{2}-132 v_{1}^{2}\right)+m_{2}^{2} \sigma_{1}^{(2)}\left(656\left(n_{12} v_{12}\right)^{2}-144\left(n_{12} v_{12}\right)\left(n_{12} v_{1}\right)\right. \\
& \left.\left.+200 v_{12}^{2}\right)+m_{1}^{2} \sigma_{2}^{(2)}\left(1124\left(n_{12} v_{12}\right)^{2}-144\left(n_{12} v_{12}\right)\left(n_{12} v_{1}\right)+436 v_{12}^{2}\right)\right] \\
& +\frac{G m_{2}}{r_{12}}\left[m_{2}^{2} \mu_{1}^{(2)}\left(252\left(n_{12} v_{1}\right)^{2}-504\left(n_{12} v_{1}\right)\left(n_{12} v_{2}\right)-\frac{387}{2}\left(n_{12} v_{2}\right)^{2}+162 v_{12}{ }^{2}-99 v_{1}^{2}\right)\right. \\
& +m_{1}^{2} \mu_{2}^{(2)}\left(-2568\left(n_{12} v_{1}\right)^{2}+5136\left(n_{12} v_{1}\right)\left(n_{12} v_{2}\right)-2946\left(n_{12} v_{2}\right)^{2}+426 v_{12}{ }^{2}-84 v_{1}^{2}\right) \\
& \left.+m_{2}^{2} \sigma_{1}^{(2)}\left(672\left(n_{12} v_{12}\right)^{2}+336 v_{12}{ }^{2}\right)+m_{1}^{2} \sigma_{2}^{(2)}\left(592\left(n_{12} v_{12}\right)^{2}+192 v_{12}{ }^{2}\right)\right]+\frac{G^{2} m_{1}^{2}}{r_{12}^{2}}\left(-\frac{2145}{7} m_{2}^{2} \mu_{1}^{(2)}\right. \\
& \left.-1008 m_{1}^{2} \mu_{2}^{(2)}\right)+\frac{G^{2} m_{1} m_{2}}{r_{12}^{2}}\left(-\frac{2581}{2} m_{2}^{2} \mu_{1}^{(2)}-1805 m_{1}^{2} \mu_{2}^{(2)}\right)+\frac{G^{2} m_{2}^{2}}{r_{12}^{2}}\left(-576 m_{2}^{2} \mu_{1}^{(2)}\right. \\
& \left.\left.-\frac{6705}{14} m_{1}^{2} \mu_{2}^{(2)}\right)\right\}+v_{1}^{i}\left\{m _ { 2 } ^ { 2 } \mu _ { 1 } ^ { ( 2 ) } \left(-144\left(n_{12} v_{1}\right)^{3}+468\left(n_{12} v_{1}\right)^{2}\left(n_{12} v_{2}\right)-720\left(n_{12} v_{1}\right)\left(n_{12} v_{2}\right)^{2}\right.\right. \\
& +360\left(n_{12} v_{2}\right)^{3}-342\left(n_{12} v_{1}\right)\left(v_{1} v_{2}\right)+360\left(n_{12} v_{2}\right)\left(v_{1} v_{2}\right)+144\left(n_{12} v_{1}\right) v_{1}^{2}-135\left(n_{12} v_{2}\right) v_{1}^{2} \\
& \left.+198\left(n_{12} v_{1}\right) v_{2}^{2}-225\left(n_{12} v_{2}\right) v_{2}^{2}\right)+m_{1}^{2} \mu_{2}^{(2)}\left(1248\left(n_{12} v_{1}\right)^{3}-3888\left(n_{12} v_{1}\right)^{2}\left(n_{12} v_{2}\right)\right. \\
& +3996\left(n_{12} v_{1}\right)\left(n_{12} v_{2}\right)^{2}-1392\left(n_{12} v_{2}\right)^{3}+9\left(n_{12} v_{1}\right)\left(v_{1} v_{2}\right)-\frac{903}{2}\left(n_{12} v_{1}\right) v_{12}{ }^{2}+492\left(n_{12} v_{2}\right) v_{12}{ }^{2} \\
& \left.-9\left(n_{12} v_{1}\right) v_{1}^{2}\right)+m_{2}^{2} \sigma_{1}^{(2)}\left(-1056\left(n_{12} v_{12}\right)^{3}+1248\left(n_{12} v_{12}\right)^{2}\left(n_{12} v_{1}\right)-576\left(n_{12} v_{12}\right)\left(n_{12} v_{1}\right)^{2}\right. \\
& -960\left(n_{12} v_{12}\right)\left(v_{1} v_{2}\right)+48\left(n_{12} v_{1}\right)\left(v_{1} v_{2}\right)+336\left(n_{12} v_{12}\right) v_{1}{ }^{2}+48\left(n_{12} v_{1}\right) v_{1}{ }^{2}+624\left(n_{12} v_{12}\right) v_{2}{ }^{2} \\
& \left.-96\left(n_{12} v_{1}\right) v_{2}^{2}\right)+m_{1}^{2} \sigma_{2}^{(2)}\left(-1664\left(n_{12} v_{12}\right)^{3}+1248\left(n_{12} v_{12}\right)^{2}\left(n_{12} v_{1}\right)-576\left(n_{12} v_{12}\right)\left(n_{12} v_{1}\right)^{2}\right. \\
& -1168\left(n_{12} v_{12}\right)\left(v_{1} v_{2}\right)+48\left(n_{12} v_{1}\right)\left(v_{1} v_{2}\right)+440\left(n_{12} v_{12}\right) v_{1}^{2}+48\left(n_{12} v_{1}\right) v_{1}^{2}+728\left(n_{12} v_{12}\right) v_{2}{ }^{2} \\
& \left.-96\left(n_{12} v_{1}\right) v_{2}^{2}\right)+\frac{G m_{1}}{r_{12}}\left[m_{2}^{2} \mu_{1}^{(2)}\left(-\frac{1209}{4}\left(n_{12} v_{1}\right)+\frac{1179}{4}\left(n_{12} v_{2}\right)\right)+m_{1}^{2} \mu_{2}^{(2)}\left(\frac{241}{4}\left(n_{12} v_{1}\right)\right.\right. \\
& \left.\left.-\frac{661}{4}\left(n_{12} v_{2}\right)\right)+m_{2}^{2} \sigma_{1}^{(2)}\left(-712\left(n_{12} v_{1}\right)+856\left(n_{12} v_{2}\right)\right)+m_{1}^{2} \sigma_{2}^{(2)}\left(-1416\left(n_{12} v_{1}\right)+1560\left(n_{12} v_{2}\right)\right)\right] \\
& +\frac{G m_{2}}{r_{12}}\left[m_{2}^{2} \mu_{1}^{(2)}\left(-378\left(n_{12} v_{1}\right)+279\left(n_{12} v_{2}\right)\right)+m_{1}^{2} \mu_{2}^{(2)}\left(714\left(n_{12} v_{1}\right)-798\left(n_{12} v_{2}\right)\right)\right. \\
& \left.\left.-1008 m_{2}^{2} \sigma_{1}^{(2)}\left(n_{12} v_{12}\right)-784 m_{1}^{2} \sigma_{2}^{(2)}\left(n_{12} v_{12}\right)\right]\right\}+v_{2}^{i}\left\{m _ { 2 } ^ { 2 } \mu _ { 1 } ^ { ( 2 ) } \left(144\left(n_{12} v_{1}\right)^{3}-468\left(n_{12} v_{1}\right)^{2}\left(n_{12} v_{2}\right)\right.\right. \\
& +720\left(n_{12} v_{1}\right)\left(n_{12} v_{2}\right)^{2}-360\left(n_{12} v_{2}\right)^{3}+342\left(n_{12} v_{1}\right)\left(v_{1} v_{2}\right)-360\left(n_{12} v_{2}\right)\left(v_{1} v_{2}\right)-144\left(n_{12} v_{1}\right) v_{1}^{2} \\
& \left.+135\left(n_{12} v_{2}\right) v_{1}^{2}-198\left(n_{12} v_{1}\right) v_{2}^{2}+225\left(n_{12} v_{2}\right) v_{2}^{2}\right)+m_{1}^{2} \mu_{2}^{(2)}\left(-1248\left(n_{12} v_{1}\right)^{3}\right. \\
& +3888\left(n_{12} v_{1}\right)^{2}\left(n_{12} v_{2}\right)-3996\left(n_{12} v_{1}\right)\left(n_{12} v_{2}\right)^{2}+1392\left(n_{12} v_{2}\right)^{3}-9\left(n_{12} v_{1}\right)\left(v_{1} v_{2}\right)+\frac{903}{2}\left(n_{12} v_{1}\right) v_{12}{ }^{2} \\
& \left.-492\left(n_{12} v_{2}\right) v_{12}^{2}+9\left(n_{12} v_{1}\right) v_{1}^{2}\right)+m_{2}^{2} \sigma_{1}^{(2)}\left(1056\left(n_{12} v_{12}\right)^{3}-1248\left(n_{12} v_{12}\right)^{2}\left(n_{12} v_{1}\right)\right. \\
& +576\left(n_{12} v_{12}\right)\left(n_{12} v_{1}\right)^{2}+960\left(n_{12} v_{12}\right)\left(v_{1} v_{2}\right)-48\left(n_{12} v_{1}\right)\left(v_{1} v_{2}\right)-336\left(n_{12} v_{12}\right) v_{1}^{2}-48\left(n_{12} v_{1}\right) v_{1}^{2} \\
& \left.-624\left(n_{12} v_{12}\right) v_{2}^{2}+96\left(n_{12} v_{1}\right) v_{2}^{2}\right)+m_{1}^{2} \sigma_{2}^{(2)}\left(1664\left(n_{12} v_{12}\right)^{3}-1248\left(n_{12} v_{12}\right)^{2}\left(n_{12} v_{1}\right)\right. \\
& +576\left(n_{12} v_{12}\right)\left(n_{12} v_{1}\right)^{2}+1168\left(n_{12} v_{12}\right)\left(v_{1} v_{2}\right)-48\left(n_{12} v_{1}\right)\left(v_{1} v_{2}\right)-440\left(n_{12} v_{12}\right) v_{1}{ }^{2}-48\left(n_{12} v_{1}\right) v_{1}^{2} \\
& \left.-728\left(n_{12} v_{12}\right) v_{2}^{2}+96\left(n_{12} v_{1}\right) v_{2}^{2}\right)+\frac{G m_{1}}{r_{12}}\left[m_{2}^{2} \mu_{1}^{(2)}\left(\frac{1209}{4}\left(n_{12} v_{1}\right)-\frac{1179}{4}\left(n_{12} v_{2}\right)\right)\right. \\
& +m_{1}^{2} \mu_{2}^{(2)}\left(-\frac{241}{4}\left(n_{12} v_{1}\right)+\frac{661}{4}\left(n_{12} v_{2}\right)\right)+m_{2}^{2} \sigma_{1}^{(2)}\left(712\left(n_{12} v_{1}\right)-856\left(n_{12} v_{2}\right)\right) \\
& \left.+m_{1}^{2} \sigma_{2}^{(2)}\left(1416\left(n_{12} v_{1}\right)-1560\left(n_{12} v_{2}\right)\right)\right]+\frac{G m_{2}}{r_{12}}\left[m_{2}^{2} \mu_{1}^{(2)}\left(378\left(n_{12} v_{1}\right)-279\left(n_{12} v_{2}\right)\right)\right.
\end{aligned}
$$




$$
\begin{aligned}
& \left.\left.\left.+m_{1}^{2} \mu_{2}^{(2)}\left(-714\left(n_{12} v_{1}\right)+798\left(n_{12} v_{2}\right)\right)+1008 m_{2}^{2} \sigma_{1}^{(2)}\left(n_{12} v_{12}\right)+784 m_{1}^{2} \sigma_{2}^{(2)}\left(n_{12} v_{12}\right)\right]\right\}\right] \\
& \left.+\frac{1}{r_{12}^{2}} n_{12}^{i}\left(-60 m_{2}^{2} \mu_{1}^{(3)}-60 m_{1}^{2} \mu_{2}^{(3)}\right)\right\}+\mathcal{O}\left(\frac{\epsilon_{\text {tidal }}}{c^{6}}\right) .
\end{aligned}
$$

The tidal part of the relative acceleration in the CoM frame, deriving from the CoM Lagrangian whose tidal part is shown in (5.4), reads

$$
\begin{aligned}
\left(a^{i}\right)_{\text {tidal }}= & -18 \frac{G^{2} m}{r^{7}} \mu_{+}^{(2)} n^{i} \\
& +\frac{1}{c^{2}}\left\{\frac { G ^ { 2 } m } { r ^ { 7 } } \left[\left((108+72 \nu) \mu_{+}^{(2)}+180 \Delta \mu_{-}^{(2)}-192 \sigma_{+}^{(2)}\right) \dot{r}^{2} n^{i}+\left(\left(-\frac{81}{2}-54 \nu\right) \mu_{+}^{(2)}-\frac{45}{2} \Delta \mu_{-}^{(2)}\right.\right.\right. \\
& \left.\left.\left.-96 \sigma_{+}^{(2)}\right) v^{2} n^{i}+\left((63-36 \nu) \mu_{+}^{(2)}-45 \Delta \mu_{-}^{(2)}+288 \sigma_{+}^{(2)}\right) \dot{r} v^{i}\right]+\frac{G^{3} m^{2}}{r^{8}}\left[(183+57 \nu) \mu_{+}^{(2)}-15 \Delta \mu_{-}^{(2)}\right] n^{i}\right\} \\
& +\frac{1}{c^{4}}\left\{\frac { G ^ { 2 } m } { r ^ { 7 } } \left[\left(\left(-3720-720 \nu+540 \nu^{2}\right) \mu_{+}^{(2)}+(-3990+900 \nu) \Delta \mu_{-}^{(2)}+(880+960 \nu) \sigma_{+}^{(2)}+160 \Delta \sigma_{-}^{(2)}\right) \dot{r}^{4} n^{i}\right.\right. \\
& +\left(\left(2472+522 \nu-288 \nu^{2}\right) \mu_{+}^{(2)}+(2724-450 \nu) \Delta \mu_{-}^{(2)}-272 \sigma_{+}^{(2)}+400 \Delta \sigma_{-}^{(2)}\right) \dot{r}^{2} v^{2} n^{i} \\
& +\left(\left(-\frac{1671}{8}-\frac{153}{2} \nu+72 \nu^{2}\right) \mu_{+}^{(2)}+\left(-\frac{1527}{8}+\frac{45}{2} \nu\right) \Delta \mu_{-}^{(2)}+(-104-96 \nu) \sigma_{+}^{(2)}-56 \Delta \sigma_{-}^{(2)}\right) v^{4} n^{i} \\
& +\left(\left(1104+36 \nu-144 \nu^{2}\right) \mu_{+}^{(2)}+(1392-180 \nu) \Delta \mu_{-}^{(2)}+(-1376-1536 \nu) \sigma_{+}^{(2)}-608 \Delta \sigma_{-}^{(2)}\right) \dot{r}^{3} v^{i} \\
& \left.+\left(\left(-\frac{633}{2}+63 \nu+36 \nu^{2}\right) \mu_{+}^{(2)}+\left(-\frac{1209}{2}+45 \nu\right) \Delta \mu_{-}^{(2)}+(872+672 \nu) \sigma_{+}^{(2)}+104 \Delta \sigma_{-}^{(2)}\right) \dot{r} v^{2} v^{i}\right] \\
& +\frac{G^{3} m^{2}}{r^{8}}\left[\left(\left(-2316+\frac{5391}{4} \nu+\frac{549}{2} \nu^{2}\right) \mu_{+}^{(2)}+\left(-2820+\frac{1665}{4} \nu\right) \Delta \mu_{-}^{(2)}+(1264+744 \nu) \sigma_{+}^{(2)}-80 \Delta \sigma_{-}^{(2)}\right) \dot{r}^{2} n^{i}\right. \\
& +\left(\left(405+\frac{887}{2} \nu-27 \nu^{2}\right) \mu_{+}^{(2)}+\left(279-\frac{135}{2} \nu\right) \Delta \mu_{-}^{(2)}+(528+216 \nu) \sigma_{+}^{(2)}-144 \Delta \sigma_{-}^{(2)}\right) v^{2} n^{i} \\
& \left.+\left(\left(336-832 \nu-114 \nu^{2}\right) \mu_{+}^{(2)}+(1092-150 \nu) \Delta \mu_{-}^{(2)}+(-1792-960 \nu) \sigma_{+}^{(2)}+224 \Delta \sigma_{-}^{(2)}\right) \dot{r} v^{i}\right] \\
& \left.+\frac{G^{4} m^{3}}{r^{9}}\left[\left(-\frac{14769}{14}-\frac{8716}{7} \nu\right) \mu_{+}^{(2)}+\left(\frac{1359}{14}+90 \nu\right) \Delta \mu_{-}^{(2)}\right] n^{i}\right\}-120 \frac{G^{2} m}{r^{9}} \mu_{+}^{(3)} n^{i}+\mathcal{O}\left(\frac{\epsilon_{\text {tidal }}}{c^{6}}\right)
\end{aligned}
$$

[1] B. P. Abbott et al. (LIGO Scientific Collaboration and Virgo Collaboration), Phys. Rev. Lett. 116, 061102 (2016), arXiv:1602.03837 [gr-qc].

[2] B. P. Abbott et al. (LIGO Scientific Collaboration and Virgo Collaboration), Phys. Rev. Lett. 119, 161101 (2017), arXiv:1710.05832 [gr-qc].

[3] J. Faber and F. Rasio, Living Rev. Relativ. 15, 8 (2012).

[4] A. Buonanno and B. Sathyaprakash, in General Relativity and Gravitation: A Centennial Perspective, edited by A. Ashtekar, B. Berger, J. Isenberg, and M. MacCallum (2015) p. 513, arXiv:1410.7832 [gr-qc].

[5] T. Mora and C. M. Will, Phys. Rev. D 69, 104021 (2004), gr-qc/0312082.

[6] E. Flanagan and T. Hinderer, Phys. Rev. D 77, 021502 (2008).

[7] T. Damour, A. Nagar, and L. Villain, Phys. Rev. D 85, 123007 (2012).

[8] M. Favata, Phys. Rev. Lett. 112, 101101 (2014), arXiv:1310.8288 [gr-qc].

[9] A. E. H. Love, Some problems of geodynamics (Cambridge U. Press, 1911).

[10] T. Hinderer, Astrophys. J. 677, 1216 (2008).

[11] T. Hinderer, B. D. Lackey, R. N. Lang, and J. S. Read, Phys. Rev. D 81, 123016 (2010).

[12] H. Fang and G. Lovelace, Phys. Rev. D 72, 124016 (2005), arXiv:1805.072660505156 [gr-qc].

[13] T. Binnington and E. Poisson, Phys. Rev. D 80, 084018 (2009), eprint arXiv:0906.1366 [gr-qc].

[14] T. Damour and A. Nagar, Phys. Rev. D 80, 084035 (2009), eprint arXiv:0906.0096 [gr-qc].

[15] B. P. Abbott et al. (LIGO Scientific Collaboration and Virgo Collaboration), Phys. Rev. X 9, 011001 (2019).

[16] B. P. Abbott et al. (LIGO Scientific Collaboration and Virgo Collaboration), Phys. Rev. X 9, 031040 (2019). 
[17] B. P. Abbott et al., "Model comparison from LIGO-Virgo data on GW170817's binary components and consequences for the merger remnant," ArXiv preprint, 1908.01012.

[18] J. Vines, T. Hinderer, and E. Flanagan, Phys. Rev. D 83, 084051 (2011), arXiv:1101.1673.

[19] D. Bini, T. Damour, and G. Faye, Phys. Rev. D 85, 124034 (2012), arXiv:1202.3565 [gr-qc].

[20] J. E. Vines and E. E. Flanagan, Phys. Rev. D 88, 024046 (2013).

[21] T. Abdelsalhin, L. Gualtieri, and P. Pani, Phys. Rev. D 98 (2018), 10.1103/PhysRevD.98.104046.

[22] B. Banihashemi and J. Vines, arXiv e-prints (2018), arXiv:1805.07266 [gr-qc].

[23] P. Landry, "Rotational-tidal phasing of the binary neutron star waveform," (2018), arXiv e-prints, 1805.01882.

[24] Q. Henry, G. Faye, and L. Blanchet, (2020), in preparation.

[25] L. Landau and E. Lifshitz, The classical theory of fields (Pergamon, Oxford, 1971).

[26] C. Misner, K. Thorne, and J. Wheeler, Gravitation (Freeman, San Francisco, 1973).

[27] E. Fermi, Atti Acad. N az. Lincei Rend. C1. Sci. Fiz. Mat. Nat. 31, 21 (1922).

[28] F. K. Manasse and C. W. Misner, Journal of Mathematical Physics 4, 735 (1963).

[29] T. Damour and A. Nagar, Phys. Rev. D 81, 084016 (2010).

[30] K. Thorne and J. Hartle, Phys. Rev. D 31, 1815 (1985).

[31] X.-H. Zhang, Phys. Rev. D 34, 991 (1986).

[32] T. Damour, M. Soffel, and C. Xu, Phys. Rev. D 43, 3273 (1991).

[33] T. Damour, P. Jaranowski, and G. Schäfer, Phys. Lett. B 513, 147 (2001), gr-qc/0105038.

[34] L. Blanchet, T. Damour, and G. Esposito-Farèse, Phys. Rev. D 69, 124007 (2004), gr-qc/0311052.

[35] L. Blanchet, T. Damour, G. Esposito-Farèse, and B. R. Iyer, Phys. Rev. D 71, 124004 (2005), gr-qc/0503044.

[36] L. Bernard, L. Blanchet, A. Bohé, G. Faye, and S. Marsat, Phys. Rev. D 93, 084037 (2016), arXiv:1512.02876 [gr-qc].

[37] L. Blanchet, G. Faye, and B. Ponsot, Phys. Rev. D 58, 124002 (1998), gr-qc/9804079.

[38] G. Schäfer, Phys. Lett. A 100, 128 (1984).

[39] J. M. Martín-García, A. García-Parrado, A. Stecchina, B. Wardell, C. Pitrou, D. Brizuela, D. Yllanes, G. Faye, L. Stein, R. Portugal, and T. Bäckdahl, "xAct: Efficient tensor computer algebra for Mathematica," (GPL 2002-2012), http://www.xact.es/.

[40] T. Damour and G. Schäfer, Gen. Rel. Grav. 17, 879 (1985).

[41] L. Blanchet, Living Rev. Relativ. 17, 2 (2014), arXiv:1310.1528 [gr-qc].

[42] L. Blanchet and G. Faye, J. Math. Phys. 42, 4391 (2001), gr-qc/0006100.

[43] V. de Andrade, L. Blanchet, and G. Faye, Class. Quant. Grav. 18, 753 (2001), gr-qc/0011063.

[44] T. Marchand, L. Bernard, L. Blanchet, and G. Faye, Phys. Rev. D 97, 044023 (2018), arXiv:1707.09289 [gr-qc].

[45] L. Blanchet and B. R. Iyer, Class. Quant. Grav. 20, 755 (2003), gr-qc/0209089.

[46] A. Le Tiec, L. Blanchet, and B. Whiting, Phys. Rev. D 85, 064039 (2012), arXiv:1111.5378 [gr-qc].

[47] E. Poisson and C. Will, Gravity: Newtonian, post-Newtonian, relativistic (Cambridge University Press, 2014 ).

[48] C. Xu, X. Wu, and G. Schäfer, Phys. Rev. D 55, 528 (1997).

[49] X. Wu, Y. He, and C. Xu, Science in China Series A: Mathematics 41, 1323 (1998).

[50] E. Racine and E. E. Flanagan, Phys. Rev. D 71, 044010 (2005), erratum: Étienne Racine, Justin E. Vines, and Éanna É. Flanagan, Phys. Rev. D 88, 089903 (2013).

[51] T. Damour, M. Soffel, and C. Xu, Phys. Rev. D 45, 1017 (1992). 